\title{
Morphometry of small recent impact craters on Mars: size and terrain dependence, short-term modification
}

\author{
W. A. Watters, ${ }^{1}$ L. M. Geiger, ${ }^{1}$ M. Fendrock, ${ }^{1}$ R. Gibson, ${ }^{1}$
}

\begin{abstract}
Most recent studies of crater morphometry on Mars have addressed large craters $(D>5 \mathrm{~km})$ using elevation models derived from laser altimetry. In the present work, we examine a global population of small $(25 \mathrm{~m} \leq D \leq 5 \mathrm{~km})$, relatively wellpreserved simple impact craters using HiRISE stereo-derived elevation models. We find that scaling laws from prior studies of large simple craters generally overestimate the depth and volume at small diameters. We show that crater rim curvature exhibits a strong diameter dependence that is well-described by scaling laws for $D<1 \mathrm{~km}$. Above this diameter, upper rim slopes begin to exceed typical repose angles and crater rims sharpen significantly. This transition is likely the result of gravity-driven collapse of the upper cavity walls during crater formation or short-term modification. In addition, we identify a tendency for small craters $(D<500 \mathrm{~m})$ to be more conical than large craters, and we show that the average cavity cross-section is well-described by a power law with exponent $\sim 1.75$ (neither conical nor paraboloidal). We also conduct a statistical comparison of crater subpopulations to illuminate trends with increasing modification and target strength. These results have important implications for describing the "initial condition" of simple crater shape as a function of diameter and geological setting, and for understanding how impact craters are modified on the martian surface over time.
\end{abstract}

\section{Introduction}

For the first three decades of the space age, studies of the distribution of 3-D impact crater shapes relied heavily on measurements from 2-D image products, using techniques such as photoclinometry (e.g., Pike and Davis [1984]) and shadow measurements (e.g., Pike [1980a]). The advent of global elevation maps from orbiting radar and laser altimeters made possible the first large-scale studies of crater shape on Venus (e.g., Herrick et al. [1997]) and Mars (e.g., Garvin and Frawley [1998], Garvin et al. [2000]), and later of Mercury (e.g., Barnouin et al. [2012], Baker et al. [2012]) and the Moon (e.g., Kalynn et al. [2013]). Most recently, elevation models of the highest resolution have been achieved by stereographic observations from high-resolution cameras on orbiting spacecraft, such as the High Resolution Stereo Camera (HRSC) on Mars Express (Jaumann et al. [2007], Heipke et al. [2007]), the High Resolution Imaging Science Experiment (HiRISE) on the Mars Reconnaissance Orbiter (McEwen et al. [2007], Kirk et al. [2008]), as well as the Lunar Reconnaissance Orbiter Camera (LROC; Robinson et al. [2010], Beyer et al. [2010]). Using these products it is possible to characterize features of impact crater shape at the ten meter scale, permitting the detailed study of even small impact craters $(D<1 \mathrm{~km}$; Stopar et al. [2012], Basilevsky et al. [2014]).

Prior studies of martian impact craters have relied heavily on Mars Orbiter Laser Altimeter (MOLA) topography (Garvin et al. [2003], Stewart and Valiant [2006], Whitehead et al. [2010], Robbins and Hynek [2012a]) to characterize scaling relationships for simple and complex craters in the range $D>5 \mathrm{~km}$. Because the simple-complex transition occurs on Mars at $D \approx 5 \mathrm{~km}$ to $D \approx 11 \mathrm{~km}$ (Robbins and Hynek [2012a]), these simple crater populations

\footnotetext{
${ }^{1}$ Department of Astronomy, Whitin Observatory,
} Wellesley College, Wellesley, Massachusetts, USA

Final submission to J. Geophysical Research: Planets, Dec. 2014 likely include the transitional "modified simple" morphology described in Pike [1988] that results from partial, gravitydriven collapse of crater walls. As such, these studies have identified relationships for the dependence of crater morphometry on size that may not be appropriate for smaller craters $(D<1 \mathrm{~km})$.

Some prior studies have sought to measure the dependence of crater depth $(d)$ on diameter $(D)$ for the freshest or deepest craters, to estimate the influence of target properties such as material strength. These studies have found that comparatively fresh craters in some lowland regions are deeper and less modified by large-scale collapse on average, implying they formed in stronger materials (Pike [1980b], Boyce et al. [2006], Stewart and Valiant [2006], Robbins and Hynek [2012a]). Whitehead et al. [2010] found that fresh craters in lava plains are deeper than craters that formed in regions dominated by sedimentary deposits. Other studies have found that craters in ice-rich polar regions also exhibit an anomalous depth distribution (Garvin et al. [2000], Fagan and Sakimoto [2012], Robbins and Hynek [2012b]). Regional variations in the simple-complex transition diameter also may suggest target materials of variable strength (Pike [1980b], Robbins and Hynek [2012a]). Finally, studies of crater morphometry have also shed light on martian surface processes and the rate of crater modification in different environments and over a range of length scales (e.g., Craddock et al. [1997], Howard [2004], Forsberg-Taylor et al. [2004], Boyce et al. [2005], Kreslavsky and Head [2006], Banks et al. [2010], Conway and Mangold [2013]).

The present study addresses these and related considerations for the small crater regime $(D<5 \mathrm{~km})$ using digital elevation models (DEMs) with the highest resolution available (down to $1 \mathrm{~m} / \mathrm{px}$ ) derived from HiRISE stereo pairs. Our goals in this study are to characterize the terrain- and diameter dependence of the shapes of relatively recent craters, to characterize how crater shape is modified over time, and to examine in detail the transition to sizes at which gravitydriven modification of the crater rim becomes an important and noticeable process.

This study presents the analysis of a database of 384 impact craters identified by reviewing the entire set of over 


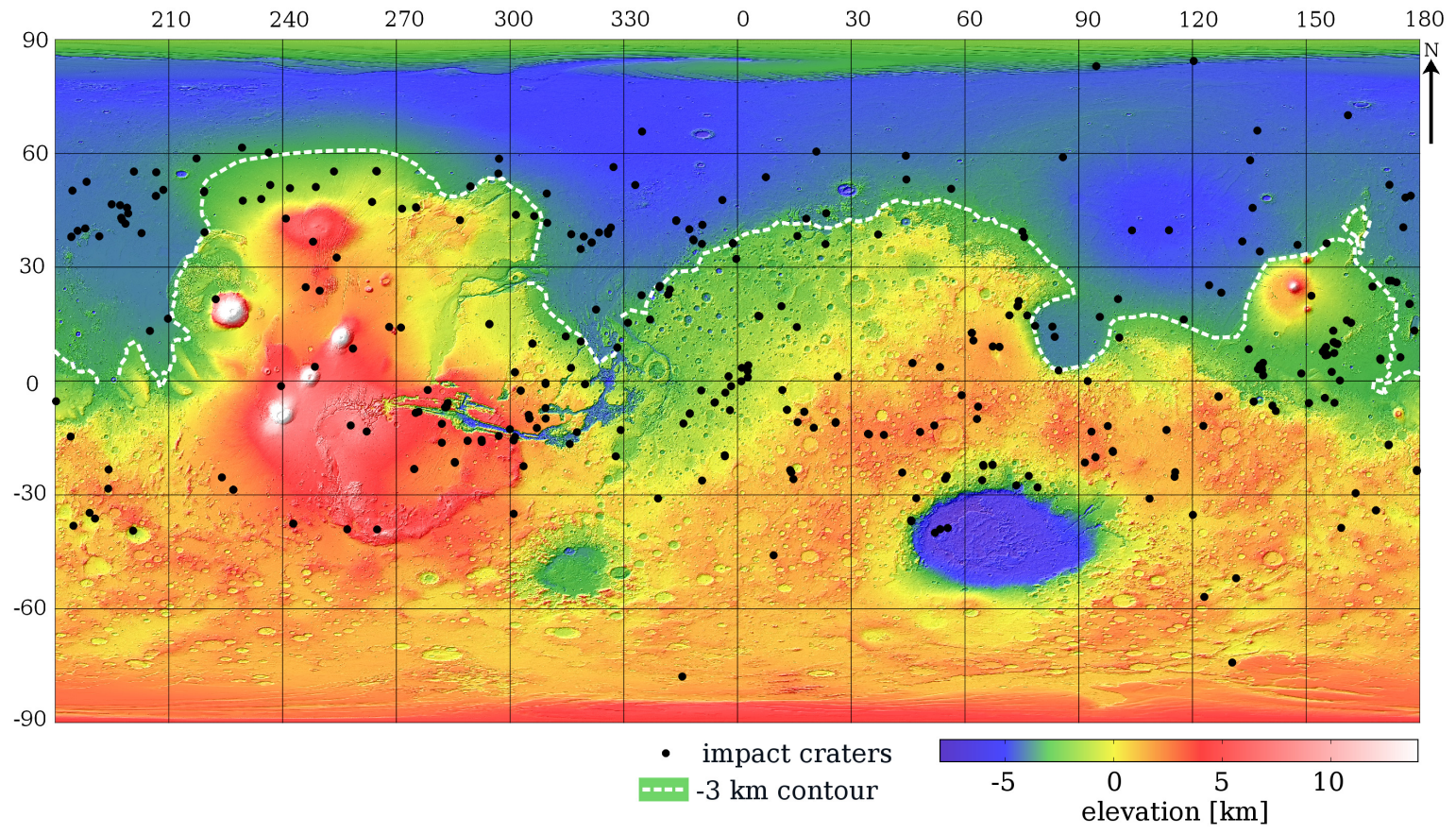

Figure 1. Population of craters in this study, plotted on the global MOLA-derived elevation model in shaded relief (Smith et al. [2001]). Note that some points overlap: not all points are individually distinguishable at this scale. A coarse trace of the contour for $-3 \mathrm{~km}$ (white dashed line) is added for reference because this elevation is used to define the "lowlands" crater subpopulation in Section 4.

3,500 stereo HiRISE image pairs available as of July 2014. The number of craters examined in this study is small compared to recent studies based on HRSC or global MOLA gridded topography, and this is the consequence mainly of two factors: (a) the global coverage by stereo HiRISE imagery remains small $(<1 \%$ of the surface as of time of writing) and (b) the production and analysis of stereo-derived DEMs is computationally expensive and time-consuming. Thankfully, statistically robust conclusions can be drawn from a smaller data set if the resolution of elevation models is relatively high. This is because measured variations more accurately reflect the intrinsic variation of the surface features, rather than variation introduced by coarse sampling of surface topography.

This paper is organized as follows. In Section 2 we describe the global population of simple impact craters under study. In Section 3 we define the relevant morphometric quantities and describe how they are measured from HiRISE-derived DEMs. Then, in Section 4 we define the subpopulations that were examined in detail and how statistical comparisons between subpopulations were carried out. This is followed in Section 5 with the measured distributions of morphometric quantities. Section 6 contains a discussion of (a) the results of statistical comparisons of subpopulations of craters modified to varying degrees, and that (b) formed in different geologic settings. We also address (c) major transitions in cavity and rim shape that are functions of diameter. Appendix A provides a description of how the extent of crater modification was assessed by inspection of orbiter imagery. Finally, Appendix B contains a detailed discussion of how the elevation models were produced, as well as a comparison of models generated using the Ames Stereo Pipeline and those published by the HiRISE Science Team and generated using BAE Systems' SOCET SET software.

\section{Crater population and database}

There is no single universally-accepted criterion for identifying fresh craters, apart from noting when they appear in new imagery. On Mars, craters that have formed in the last decade tend to be small ( $D<35 \mathrm{~m}$; Daubar et al. [2013]) and are poorly-resolved where present in a handful of stereo HiRISE observations. When bounds cannot be placed on the timing of crater formation, a set of criteria are often used to identify recent craters, including: the relation of depth and rim height to diameter (as in Stewart and Valiant [2006]); the qualitatively-assessed "sharpness" of rim crests (e.g., Barlow [2004]); the absence of impact craters on ejecta (e.g., Kalynn et al. [2013]); and the visibility of continuous and rayed ejecta (e.g., Tornabene et al. [2006]).

Unfortunately, none of these criteria can be applied in an entirely consistent way when considering a large range of crater diameters. This is partly because the processes that modify the overall shape and appearance of impact craters occur at different rates for craters of different sizes. For example, the rims and ejecta deposits of large craters are likely to endure much longer than the rims of small craters. The appearance of ejecta surrounding small craters can change dramatically following dust storms (Geissler et al. [2010]) that have almost no effect on the visibility of ejecta surrounding larger craters.

Our global database of 384 impact craters was assembled through browsing HiRISE stereo images and selecting craters that exhibit one or more of the following hallmarks of good preservation: (a) an apparently deep cavity or (b) ejecta that are visible because of a contrast in brightness or texture. In all cases these qualitative criteria were used to select craters that were relatively well-preserved when compared with other craters in the same image (if any). As a result, the database includes craters in a range of modification states, from extremely fresh to significantly modified.

DEMs were generated for all the craters selected on this basis $(N=384)$, but most of the results reported in Section 

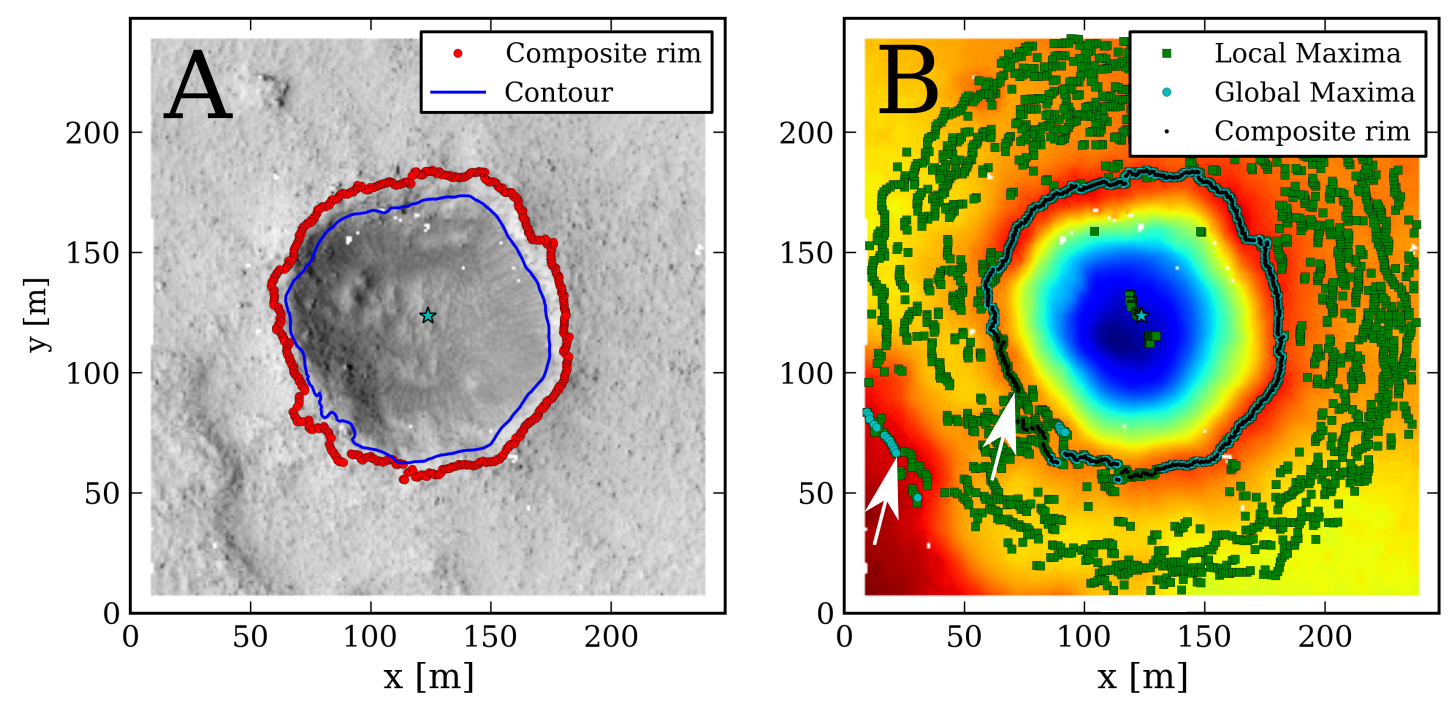

Figure 2. (A) Rim trace (red) derived from stitching segments of global and local maxima along radial elevation profiles sampled from the DEM at right. Also shown is an elevation contour at the rim's lowest elevation (blue). (B) Plot of the global maxima (cyan circles) measured from radial profiles of the elevation model of the simple impact crater at left in (A), as well as local maxima (green squares) and the final composite rim trace $\mathbf{T}_{\mathbf{1}}$ (black circles). Highest elevations are colored in red, lowest in blue. Arrows point to a discontinuity where local maxima were used to bridge a gap. The $\mathrm{x}$ and $\mathrm{y}$ axes indicate position in meters with respect to a convenient local origin.

5 are derived from subpopulations that have undergone additional screening to remove the most degraded craters (see Section 4). To avoid significant contamination from secondaries, we have also avoided or removed from consideration all craters that occur inside or within several kilometers of thermally-distinct rays of the fresh complex craters Gratteri and Zunil (Tornabene et al. [2006]). We cannot be certain that the population we have studied does not contain some very distant secondaries.

The database of 384 craters is included with the online Supplementary Materials. These range in diameter from 25 $\mathrm{m}$ to $5.2 \mathrm{~km}$. Roughly $27 \%$ of these craters are located in the northern lowlands (lat $>0^{\circ}$, elevation $<-3 \mathrm{~km}, \sim 22 \%$ of planetary surface area) and roughly $15 \%$ are located at high latitudes (|lat. $\mid>45^{\circ}, \sim 29 \%$ of planetary surface area). Their global distribution is plotted in Figure 1. For each crater, we supply a precise estimate of the latitude and longitude, the HiRISE stereo pair observation IDs, estimates of all quantities discussed in this study along with uncertainties, as well as a set of qualitative attributes that includes, among others: distinctive appearance of ejecta via texture or brightness or raised margins in HiRISE imagery; presence of craters on ejecta; step or bench in crater walls; presence of thermally-distinct ejecta (i.e., visible in nighttime Thermal Emission Imaging System (THEMIS) imagery (Christensen et al. [2004])); whether ejecta have lobate margins; whether rim indicates signs of mass wasting or gully formation.

\section{Measurements of crater shape}

The measurements presented in this study relate to (a) the shape of the vertical cross-section of simple impact craters as well as (b) the volume of crater cavities. We begin with digital elevation models (DEMs) that were generated in-house using the Integrated Software for Imagers and Spectrometers (ISIS 3.4) developed by the United States Geological Survey (Anderson et al. [2004], Becker et al. [2007]) in conjunction with the Ames Stereo Pipeline (ASP 2.0; Broxton and Edwards [2008], Moratto et al. [2010]) from stereo image pairs acquired by the HiRISE camera on-board the
Mars Reconnaissance Orbiter (McEwen et al. [2007]). Our DEMs range in resolution from approximately $1 \mathrm{~m} / \mathrm{px}$ to 15 $\mathrm{m} / \mathrm{px}$. At maximum resolution, a $4 \times 4$ square of HiRISE pixels corresponds to one pixel in our DEMs. The process used to make these models is described in Appendix B, where they are also compared to DEMs published by the HiRISE Science Team and generated using the methods described in Kirk et al. [2008]. On average, the pixel-to-pixel disagreement in elevation is found to be $<0.5 \mathrm{~m}$.

\subsection{Rim trace and radial profiles}

The first step in our process is to locate craters manually and make a rough estimate of radius by selecting an approximate center and two rim points. Once DEMs are

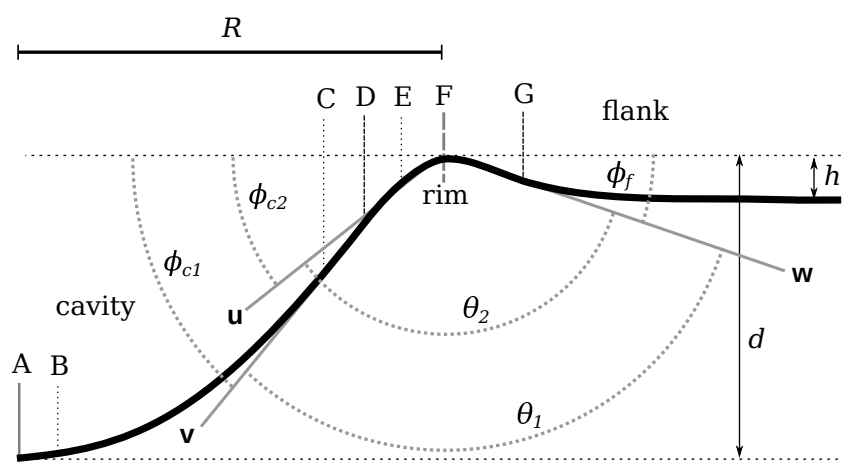

Figure 3. Artificial radial elevation profile with labeled quantities and reference positions used in Table 1. The reference positions $\mathrm{A}$ and $\mathrm{B}$ are separated by radial distance $0.1 R$, as are $\mathrm{C}$ and $\mathrm{D}, \mathrm{D}$ and $\mathrm{E}$, as well as $\mathrm{E}$ and $\mathrm{F}$. F and $\mathrm{G}$ are separated by a distance $0.2 R$. The line $\mathrm{u}$ extends a line fitted to the rim between positions $\mathrm{D}$ and $\mathrm{F}$, while the line $\mathrm{v}$ is fitted to the profile from $\mathrm{C}$ to $\mathrm{E}$, and the line $\mathrm{w}$ is fitted from $\mathrm{F}$ to $\mathrm{G}$. The diameter $D=2 R$ is the "rim diameter" of Turtle et al. [2005], also sometimes called the "rim crest diameter". 
Table 1. Quantities computed from crater DEMs. Type 1 quantities are measured from individual radial profiles and then averaged. Type 2 quantities are measured using the rim trace or 3-D cavity surface or both. Refer to Figure 3 for the radial positions $\mathrm{A}-\mathrm{G}$ and the lines $\mathrm{u}, \mathrm{v}$, and $\mathrm{w}$.

\begin{tabular}{|c|c|c|c|}
\hline Symbol & Type & Name & Definition \\
\hline$\xi_{c}$ & 1 & upper cavity wall radius-of-curvature & Radius of circle fitted to the profile from $\mathrm{D}$ to $\mathrm{F}$. \\
\hline$\xi_{f}$ & 1 & upper flank radius-of-curvature & Radius of circle fitted to the profile from $\mathrm{F}$ to $\mathrm{G}$. \\
\hline$\ell_{c}$ & 1 & cavity rim decay length & $\begin{array}{l}\text { Distance from rim to position within cavity where slope is } \\
1 / 2 \text { the maximum slope along cavity profile. }\end{array}$ \\
\hline$\ell_{f}$ & 1 & flank rim decay length & $\begin{array}{l}\text { Distance from rim to position within flank where slope is } 1 / 2 \\
\text { the maximum slope along flank profile. }\end{array}$ \\
\hline$\alpha_{c}$ & 1 & cavity shape exponent & Exponent of power-law fit to radial profile from B to E. \\
\hline$\phi_{c 1}$ & 1 & middle cavity wall slope angle & Slope angle of line fitted from $\mathrm{C}$ to E (line v). \\
\hline$\phi_{c 2}$ & 1 & upper cavity wall slope angle & Slope angle of line fitted from D to F (line u). \\
\hline$\phi_{f}$ & 1 & flank slope angle & Slope angle of line fitted from $\mathrm{F}$ to $\mathrm{G}$ (line w). \\
\hline$\theta_{1}$ & 1 & lower rim span & $180^{\circ}-\left(\phi_{c 1}+\phi_{f}\right) ;$ the angle subtended by lines $\mathrm{v}$ and $\mathrm{w}$. \\
\hline$\theta_{2}$ & 1 & upper rim span & $180^{\circ}-\left(\phi_{c 2}+\phi_{f}\right) ;$ the angle subtended by lines $\mathrm{u}$ and $\mathrm{w}$. \\
\hline$h$ & 1 & average rim height & $\begin{array}{l}\text { Average height of rim above elevation minimum in radial } \\
\text { profile of surrounding terrain. }\end{array}$ \\
\hline$D$ & 2 & diameter & Average rim crest diameter. \\
\hline$d$ & 2 & average rim-to-floor depth & Average rim elevation minus minimum cavity elevation. \\
\hline$V$ & 2 & cavity volume & $\begin{array}{l}\text { Cavity volume below elevation that is average of (i) lowest } \\
\text { elevation along the rim and (ii) estimated average elevation } \\
\text { of surroundings. }\end{array}$ \\
\hline$i_{\mathrm{M}}$ & & crater modification index & $\begin{array}{l}\text { Index based on qualitative observations of crater preserva- } \\
\text { tion (see Appendix A). }\end{array}$ \\
\hline
\end{tabular}

generated (see Appendix B), the next step is to extract the 3 -D trace of the crater rim crest (the "rim trace"). This is initially accomplished in an automatic way using a collection of Python scripts developed in-house (Geiger [2013]). First, the elevations of two circles are sampled at distances $3 R$ and $3.5 R$ from the crater center, using the provisional, manually-selected radius $R$. The plane fitted to these points is representative of the surrounding surface and is subtracted from the DEM. Then, 512 evenly-spaced radial profiles are sampled from the DEM to a distance $2 R$ from the center (for later estimates of rim height, profiles are measured to $4 R$ ). We sample $2^{9}=512$ profiles because a number $2^{n}$ is computationally expedient, and $2^{10}=1024$ profiles would significantly oversample a large fraction of the craters in our database. (As discussed later in this section, we use only non-overlapping radial profiles to measure shape parameters from the crater rim and flank.) The position of the global maximum elevation along each profile is marked as candidate rim point. A plane is fitted to these candidate points and subtracted from the DEM, and the positions of global elevation maxima are selected a second time. These points make up the candidate rim trace $\mathbf{T}_{\mathbf{0}}$.

This candidate rim trace invariably contains discontinuities and will sometimes overshoot the true crater rim (e.g., finding a more distal ridge or the rim crest of a nearby crater). For this reason, our process also identifies local maxima (see Figure 2) and then assembles a composite crater rim using these local maxima to fill gaps in $\mathbf{T}_{\mathbf{0}}$. An example of a rim automatically mended in this way is shown in Figure 2 . The set of points in this composite rim trace is called $\mathbf{T}_{\mathbf{1}}$. Finally, a user manually reviews the rims extracted via the automatic process. Where major discontinuities persist, we use an in-house interface to mend the rim manually by choosing points identified by the software as local maxima or major breaks in slope. This manual intervention is required in less than $10 \%$ of cases.

Because the surrounding terrain is often complex, we cannot sample and subtract the surrounding pre-impact surface in an automatic and self-consistent way. For this reason, we take an approach similar to that described in Vincent et al. [2013], where crater shapes are measured on the heavilycratered asteroid Vesta. In that case, the straight line connecting crater rims in cross-sectional profiles was subtracted from the profile as a whole, in order to measure crater depth. Instead, we subtract the plane fitted to the 3-D rim trace $\mathbf{T}_{\mathbf{1}}$, while acknowledging that this is likely in most cases to be similar but not exactly parallel to the local equipotential surface (Vijayan et al. [2013]). The cavity volume is potentially somewhat affected by this choice because, as discussed in Section 3.2, an elevation contour is used to define a bounding surface. Subtracting the rim-fitted plane hence ensures that we measure the largest volume encompassed by the crater rim. The measurement of rim height $h$ is not affected by this choice because it is measured from individual radial profiles that are separately detrended using elevations sampled at a distance $>3 R$.

A circle is fitted to the $2-\mathrm{D}$ projection of $\mathbf{T}_{\mathbf{1}}$ to measure the diameter and geometric center, $P_{0}$. Then, 512 radial profiles are sampled with respect to $P_{0}$ at the same density as the DEM post spacing and with linear interpolation. An example artificial radial cross-section is shown in Figure 3, with marked reference positions that delimit the radial span over which shape quantities are calculated in the crater cavity and flanks. These reference positions are separated by a radial distance $0.1 R$; e.g., position $\mathrm{D}$ is $0.2 R$ from position $\mathrm{F}$ (from the rim). The quantities computed from radial profiles are defined in Table 1. These include: the power-law exponent of the cavity cross-section $\left(\alpha_{c}\right)$; the rim-to-floor depth $(d)$; slope angles measured from the middle cavity wall, upper cavity wall, and flank surface $\left(\phi_{c 1}, \phi_{c 2}, \phi_{f}\right)$; rim height $(h)$; radius-of-curvature of cavity-facing and flankward rim surfaces $\left(\xi_{c}, \xi_{f}\right)$, and the "rim decay" length scales $\left(\ell_{c}, \ell_{f}\right.$ ) . Subscripts $c$ and $f$ indicate quantities measured in the cavity and flank, respectively, which are separated by the rim at position $\mathrm{F}$ in Figure 3.

The Ames Stereo Pipeline 2.0 does not supply absolute uncertainties for individual elevations, and so these cannot be included in the analysis. Profile fits were therefore computed assuming an uncertainty comparable to the locallysampled topographic variation. To report the estimated value and uncertainty of morphometric parameters that are characteristic of the crater as whole, we measure the azimuthal variation in a way similar to Baker et al. [2012] 
(where median values were reported) for each quantity in Table 1 . We use only non-overlapping radial profiles to estimate rim and flank parameters: i.e., we discard redundant, overlapping profiles. For the only full-cavity shape parameter, $\alpha_{c}$, we allow overlap up to half the crater radius from the crater center (to avoid discarding the vast majority of radial profiles, since overlap near the center is common).

For all parameters, we report azimuthally-averaged values and the uncertainties of these averages: e.g., we compute $\sigma / \sqrt{n}$ for standard deviation $\sigma$ and the number of nonoverlapping radial profiles $n$, which depends on the crater size and DEM resolution (even for $\alpha_{c}, n$ is the number of profiles that do not overlap at all). Where measurements from individual profiles have an associated uncertainty (such as the uncertainty of a fit), we report the error-weighted azimuthal mean and the error-weighted uncertainty of this mean. Tables of estimated values and uncertainties for all morphometric quantities addressed in this paper and all craters in our database can be found in the online supplementary materials.

The high resolution of HiRISE-derived elevation models makes it possible to measure subtle shape characteristics such as rim sharpness or "roundness". This property is quantified here in several ways. First, the upper cavity wall radius-of-curvature $\left(\xi_{c}\right)$ is the estimated radius of a circle fitted to the cavity wall profile between radial positions D and F (Figure 3). For a relatively rounded upper cavity wall, this value will be smaller. By contrast, a flat upper cavity wall will have a near infinite radius with a large uncertainty.

Second, the "rim decay length" $\left(\ell_{c}\right)$ is the distance over which the slope of the radial profile, measured while marching toward the cavity center, changes from zero (at the rim) to one-half the maximum slope of the cavity wall. The exact position of the maximum slope is very sensitive to very small variations in local slope. By contrast, the point at which half this value is reached exhibits far less variation within a single crater. A relatively sharp rim with a flat upper cavity wall will exhibit a relatively short rim decay length. By contrast, a relatively rounded upper cavity wall, in which the slope changes more gradually, will have a relatively large rim decay length. We also measure $\ell_{f}$ and $\xi_{f}$, the counterparts to $\ell_{c}$ and $\xi_{c}$ measured on the crater flank from $\mathrm{F}$ to $\mathrm{G}$ in Figure 3.

Third, we measure the angle subtended by the cavity- and flankward slopes, here called the "rim span". The value of $\theta_{1}$ is the angle subtended by the flankward slope (line w in Figure 3) and the middle cavity wall slope (line v in Figure 3 ), while $\theta_{2}$ is the angle subtended by the flankward slope and the upper cavity wall slope (line $u$ in Figure 3 ). As the rim span decreases, the rim becomes "narrower" in the sense of being flanked by steeper slopes.

In each crater DEM, some fraction of elevations are discarded by the ASP or by the post-processing steps described in Appendix B. For this reason, the radial profile quantities ("Type 1") are computed for a given radial profile only if there exists more than a threshold fraction of valid computed elevations in the relevant range of radial positions (see Table 1). This threshold fraction is $75 \%$ or larger in the case of most of the quantities reported. We have not computed shape characteristics from radial profiles using interpolated elevations.

\subsection{Crater cavity volume}

In addition to quantities measured from radial elevation profiles, we estimate the volume of the crater cavity. First we compute two elevation contours that define bounding surfaces for the numerical integration: (a) at the lowest elevation along the rim and (b) at the approximate elevation of the surrounding topography, densely sampled in two circles at distances $3 R$ and $3.5 R$ from the crater center (beyond the influence of ejecta). The volume we report is the average volume computed using these two bounding surfaces, and the reported uncertainty is one-half the difference between them. This average is considered preferable because the surrounding topography is often complex, complicating the estimate of the pre-impact surface elevation. Also, our main interest is to measure the dependence of volume on crater diameter: we are not concerned with comparing cavity and ejecta volumes as other studies have done (e.g., Black and Stewart [2008]). We checked our volume estimates using artificiallygenerated paraboloid craters of known volume, and verified that the uncertainty of the calculation is much smaller than the difference in estimates based on bounding surfaces (a) and $(b)$.

\section{Subpopulation comparisons}

Throughout this work we will refer to a number of subsets or subpopulations of special interest. These have been identified for special consideration and comparison, to examine the effects of differences in diameter, extent of modification, and target material properties. These subpopulations are defined in Table 2.

We begin by assigning the craters in our database to categories according to whether they are relatively wellpreserved or significantly modified. To this end, all craters were assigned a "modification index", $i_{M}$, defined in Appendix A and based on visual inspection of craters in HiRISE and THEMIS night-IR images. According to this scheme, craters with cavity deposits, cratered cavities and ejecta, and degraded ejecta receive higher scores on a scale from -2 to +6 . We then subdivide our database into three subpopulations based only on $i_{M}$, and then a further three subpopulations based on a combination of $i_{M}$, the rim height / crater

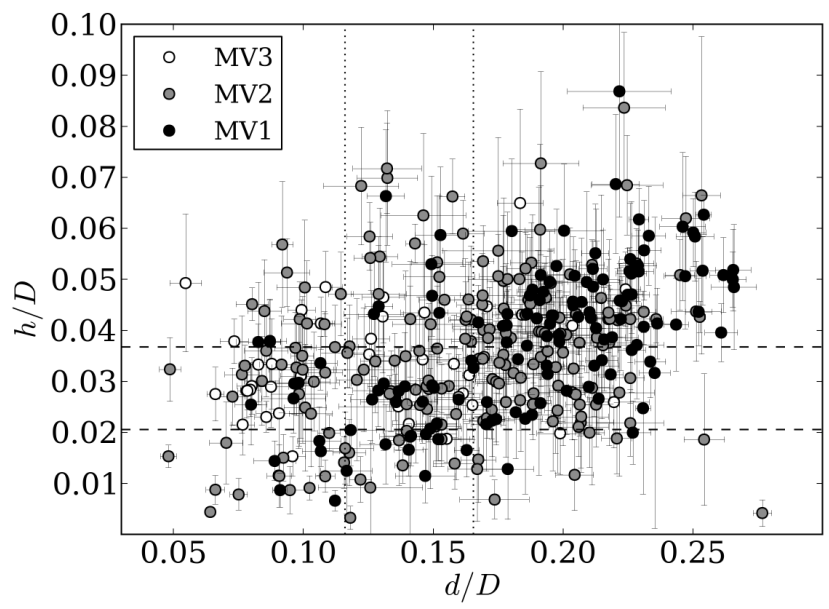

Figure 4. Diameter-normalized rim height $(h / D)$ versus rim-to-floor depth $(d / D)$. Error bars represent propagated standard deviation rather than uncertainty of mean values (which are generally smaller than the symbol size because results are averaged from a large number of radial profiles). On the basis of visual inspection, using an index defined in Appendix A, white points represent the most modified craters (MV3) and black points the least modified (MV1), with gray points in between (MV2). Only for MV1 are rim height and crater depth significantly correlated (Pearson correlation coefficient $\rho=0.58$ ). Dashed and dotted lines represent mean values and (mean - $\sigma$ ), used to construct a semi-quantitative criterion for assessing preservation (see LM, MM, and HM in Table 2). $(N=372)$ 
Table 2. Definitions of crater subpopulations addressed in this study.

\begin{tabular}{|c|c|c|}
\hline Name & $N$ & Definition \\
\hline modif. vis. level 1 (MV1) & 139 & $\begin{array}{l}i_{\mathrm{M}} \leq 0 \text { : least modified craters, assessed by visual inspection (HiRISE, } \\
\text { THEMIS) }\end{array}$ \\
\hline modif. vis. level 2 (MV2) & 204 & $\begin{array}{l}0<i_{\mathrm{M}} \leq 3: \text { craters somewhat modified, assessed by visual inspection } \\
\text { (HiRISE, THEMIS) }\end{array}$ \\
\hline modif. vis. level 3 (MV3) & 41 & $\begin{array}{l}i_{\mathrm{M}}>3 \text { : craters significantly modified, assessed by visual inspection } \\
\text { (HiRISE, THEMIS) }\end{array}$ \\
\hline moderately modified (MM) & 261 & $\begin{array}{l}i_{\mathrm{M}} \leq 3, d / D>\langle d / D\rangle_{\mathrm{avg}}-\sigma_{d / D}=0.116, h / D>\langle h / D\rangle_{\mathrm{avg}}-\sigma_{h / D}= \\
0.021\end{array}$ \\
\hline highly modified (HM) & 123 & $\begin{array}{l}\text { All craters that do not belong to the "moderately modified" subpopu- } \\
\text { lation. }\end{array}$ \\
\hline least modified (LM) & 70 & $i_{\mathrm{M}} \leq 0, d / D>\langle d / D\rangle_{\mathrm{avg}}=0.16, h / D>\langle h / D\rangle_{\mathrm{avg}}=0.036$ \\
\hline large craters I & 97 & $D \geq 1 \mathrm{~km}$ \\
\hline large craters II & 126 & $D \geq 500 \mathrm{~m}$ \\
\hline small craters I & 287 & $D<1 \mathrm{~km}$ \\
\hline small craters II & 258 & $D<500 \mathrm{~m}$ \\
\hline lavas & 85 & $\begin{array}{l}\text { Formed in lava flows or flood lavas; superset of units } \mathrm{AHv}, \mathrm{eHv}, \mathrm{lAv} \text {, } \\
\mathrm{lAvf} \text { and } \mathrm{lHv} \text { in Tanaka et al. [2014]. }\end{array}$ \\
\hline volcanics & 110 & $\begin{array}{l}\text { Formed in region dominated by lava, pyroclastic, or volcaniclastic flows; } \\
\text { superset of geologic units AHv, Av, Ave, eHv, Hve, lAv, lAvf, lHv, lHvf, } \\
\text { lNv, Nhe, Nve in Tanaka et al. [2014]. }\end{array}$ \\
\hline coarse deposits & 27 & $\begin{array}{l}\text { Formed in units that contain coarse-grained deposits: crater interiors } \\
\text { and ejecta, drop moraines, mass wasting products, catastrophic debris } \\
\text { flow deposits; superset of units Aa, AHi, Ht, Hto, lAa, lHt in Tanaka } \\
\text { et al. }[2014] \text {. }\end{array}$ \\
\hline pure coarse deposits & 18 & $\begin{array}{l}\text { Formed in units dominated by coarse-grained deposits: superset of units } \\
\text { Aa and lAa in Tanaka et al. [2014]. }\end{array}$ \\
\hline fine deposits & 65 & $\begin{array}{l}\text { Formed in units that contain fine-grained deposits: airfall, fluvial, la- } \\
\text { custrine deposits and aeolian sand plains; superset of units AHtu, ANa, } \\
\mathrm{Ap}, \mathrm{Apu}, \mathrm{eAb}, \mathrm{Hpu}, \mathrm{Ht}, \mathrm{Hto}, \mathrm{Htu}, \mathrm{lApd}, \mathrm{Hb}, \mathrm{HHl}, \mathrm{Ht}, \mathrm{mAl} \text { in Tanaka } \\
\text { et al. }[2014] \text {. }\end{array}$ \\
\hline Vastitas Borealis & 45 & $\begin{array}{l}\text { Formed in the Vastitas Borealis unit } \mathrm{lHl} \text { (fine deposits over flood lavas), } \\
\text { defined in Tanaka et al. [2014]. }\end{array}$ \\
\hline impact unit & 83 & $\begin{array}{l}\text { Formed in moderately to well-preserved crater or crater ejecta, assessed } \\
\text { by visual inspection (HiRISE, THEMIS), or formed in unit AHi as de- } \\
\text { fined in Tanaka et al. [2014]. }\end{array}$ \\
\hline high latitude & 57 & Located at $\mid$ latitude $\mid>45^{\circ}$. \\
\hline low latitude & 327 & Located at $\mid$ latitude $\mid<45^{\circ}$ \\
\hline highlands & 269 & $\begin{array}{l}\text { Located at elevation }>-3 \mathrm{~km} \text { (MOLA gridded elevation model, Smith } \\
\text { et al. }[2001]) \text {. }\end{array}$ \\
\hline lowlands & 109 & $\begin{array}{l}\text { Located at latitude }>0^{\circ} \text {, elevation } \leq-3 \mathrm{~km} \text { (MOLA gridded elevation } \\
\text { model, Smith et al. }[2001]) \text {. }\end{array}$ \\
\hline lobate ejecta & 33 & Crater ejecta exhibit lobate distal margins. \\
\hline
\end{tabular}

diameter ratio $(h / D)$, and the crater depth/diameter ratio $(d / D)$. The categories based on $i_{M}$ alone are three levels of "modification assessed by visual inspection," abbreviated MV1, MV2, and MV3. MV3 is the subpopulation with the most modified craters, having the largest values of $i_{M}$; MV1 has the least modified craters with the lowest values of $i_{M}$.

A plot of $h / D$ versus $d / D$ is shown in Figure 4 with each crater labeled according to its membership in MV1, MV2, and MV3. Only for MV1 are rim height and crater depth significantly correlated, with a Pearson correlation coefficient of $\rho=0.58$, whereas $\rho=0.25$ for craters in MV2 and $\rho=0.17$ for craters in MV3. That is, rim height and crater depth are significantly correlated for relatively unmodified craters, but this correlation decreases as craters accumulate more visual hallmarks of modification. This suggests that modification can affect cavities and rims to varying degrees, and that otherwise related morphometric parameters may become largely decoupled with increasing modification. It is also worth noting that a significant fraction of apparently fresh craters exhibit a wide range of rim heights and crater depths. A discussion of detailed statistical comparisons between these subpopulations (MV1-MV3) is saved for Section 6.1 .

We expect erosion to flatten crater rims, widen crater diameters, and fill-in crater cavities, with a modification trend indicated by $h / D \rightarrow 0$ and $d / D \rightarrow 0$. Based on this assumption, we also group craters in a semi-quantitative sense as follows. "Moderately modified craters" (MM) are all craters that satisfy the following conditions: (a) do not belong to MV3 (the most modified, as assessed by visual inspection); (b) have $h / D$ that exceeds the average of $h / D$ minus its standard deviation (lower horizontal dashed line in Figure 4 ); and (c) have $d / D$ that exceeds the average of $d / D$ minus one standard deviation (left-most vertical dotted line in Figure 4). "Highly modified" (HM) craters are all those craters that do not belong to "moderately modified" (MM). Finally, "least modified" craters (LM) form a subset of "moderately modified", and consist of all craters in MV1 (well-preserved, as assessed by visual inspection alone) and which have $h / D$ 
Table 3. Table of subpopulation comparisons using the KS test: quantities pertaining to cavity shape and dimensions. In all cases (including results in Tables 4 and 5), $n_{B} n_{A} /\left(n_{A}+n_{B}\right)>4$ and $p<0.05$. Note that $\sigma_{A}$ and $\sigma_{B}$ represent one standard deviation of quantities $q_{A}$ and $q_{B}$, respectively. The "Filter" column supplies the subpopulation used to filter craters before the comparison was made. For example, if the filter is MM, subpopulation A is "lavas", and subpopulation B is "impact unit", then the KS test was used to compare moderately modified craters (MM) that formed in lavas to moderately modified craters that formed in the impact unit. See Table 2 for subpopulation definitions.

\begin{tabular}{|c|c|c|c|c|c|c|c|c|}
\hline$q$ & Filter & A & $\mathrm{B}$ & $\left(n_{A}, n_{B}\right)$ & $q_{A} \pm \sigma_{A}$ & $q_{B} \pm \sigma_{B}$ & $p$ & ref. ID \\
\hline \multirow[t]{12}{*}{$\alpha_{c}$} & none & modif. vis. L1 & modif. vis. L3 & $(130,40)$ & $1.60 \pm 0.40$ & $1.88 \pm 0.36$ & 0.0002 & $\mathrm{~m} 1$ \\
\hline & none & moderately modif. & highly modified & $(248,121)$ & $1.61 \pm 0.40$ & $1.73 \pm 0.42$ & 0.0008 & $\mathrm{~m} 2$ \\
\hline & LM & large $(D \geq 1 \mathrm{~km})$ & small $(D<1 \mathrm{~km})$ & $(33,23)$ & $2.04 \pm 0.46$ & $1.60 \pm 0.28$ & 0.0061 & a1 \\
\hline & $\mathrm{LM}$ & large $(D \geq 500 \mathrm{~m})$ & $\operatorname{small}(D<500 \mathrm{~m})$ & $(37,19)$ & $2.00 \pm 0.45$ & $1.58 \pm 0.30$ & 0.0044 & $\mathrm{a} 2$ \\
\hline & MM & large $(D \geq 1 \mathrm{~km})$ & small $(D<1 \mathrm{~km})$ & $(77,134)$ & $1.90 \pm 0.43$ & $1.66 \pm 0.41$ & $<10^{-4}$ & a3 \\
\hline & MM & large $(D \geq 500 \mathrm{~m})$ & small $(D<500 \mathrm{~m})$ & $(92,119)$ & $1.88 \pm 0.43$ & $1.65 \pm 0.40$ & $<10^{-4}$ & $\mathrm{a} 4$ \\
\hline & MV1 & large $(D \geq 1 \mathrm{~km})$ & small $(D<1 \mathrm{~km})$ & $(40,52)$ & $1.99 \pm 0.47$ & $1.56 \pm 0.34$ & $<10^{-4}$ & a5 \\
\hline & MV1 & large $(D \geq 500 \mathrm{~m})$ & $\operatorname{small}(D<500 \mathrm{~m})$ & $(47,45)$ & $1.92 \pm 0.46$ & $1.56 \pm 0.36$ & 0.0001 & a6 \\
\hline & MM & impact unit & elsewhere & $(40,171)$ & $1.61 \pm 0.43$ & $1.78 \pm 0.42$ & 0.0126 & s1 \\
\hline & MM & fine deposits & elsewhere & $(41,170)$ & $1.83 \pm 0.34$ & $1.73 \pm 0.44$ & 0.0147 & i1 \\
\hline & MV1 & lobate ejecta & not lobate & $(12,80)$ & $1.88 \pm 0.31$ & $1.73 \pm 0.47$ & 0.0474 & $\mathrm{i} 2$ \\
\hline & MV1 & Vastitas Bor. & elsewhere & $(10,82)$ & $1.86 \pm 0.27$ & $1.73 \pm 0.47$ & 0.0182 & i3 \\
\hline \multirow[t]{8}{*}{$d / D$} & none & modif. vis. L1 & modif. vis. L2 & $(139,204)$ & $0.18 \pm 0.04$ & $0.16 \pm 0.04$ & 0.0002 & $\mathrm{~m} 3$ \\
\hline & none & modif. vis. L1 & modif. vis. L3 & $(139,41)$ & $0.18 \pm 0.04$ & $0.12 \pm 0.04$ & $<10^{-4}$ & $\mathrm{~m} 4$ \\
\hline & MM & lavas & elsewhere & $(62,199)$ & $0.19 \pm 0.03$ & $0.18 \pm 0.03$ & 0.0218 & $\mathrm{~s} 2$ \\
\hline & MM & volcanics & elsewhere & $(76,185)$ & $0.19 \pm 0.03$ & $0.18 \pm 0.03$ & 0.0087 & s3 \\
\hline & MM & impact unit & lavas & $(55,62)$ & $0.17 \pm 0.03$ & $0.19 \pm 0.03$ & 0.0086 & s4 \\
\hline & MV1 & impact unit & elsewhere & $(29,110)$ & $0.17 \pm 0.03$ & $0.18 \pm 0.04$ & 0.0260 & s5 \\
\hline & MV1 & pure coarse & elsewhere & $(8,131)$ & $0.14 \pm 0.03$ & $0.18 \pm 0.04$ & 0.0222 & s6 \\
\hline & MM & lobate ejecta & not lobate & $(32,229)$ & $0.20 \pm 0.02$ & $0.18 \pm 0.03$ & 0.0072 & i4 \\
\hline
\end{tabular}

and $d / D$ that exceed the average values of these quantities for the population as a whole. Many of the analyses conducted in later sections address $\mathrm{MM}(N=261)$, which includes LM $(N=70)$ and excludes all of the most heavily modified craters (HM, $N=123$ ).

The second major category of subpopulations relate to the terrain type in which craters formed. To assign craters to geological settings, we made use of the global geological map of Tanaka et al. [2014]. Map units were assigned to the categories listed in Table 2: fine-grained deposits, coarsegrained deposits, impact unit, lavas, and "volcanics". Units made up of a mixture of more than two distinctive geological materials were not considered in this study, and craters that formed in those units were not included in the geological subpopulation comparisons discussed below. The "impact unit" subpopulation consists of craters in unit AHi of Tanaka et al. [2014], along with all craters that we observed to reside inside the cavity or continuous ejecta blanket of moderately well-preserved impact craters.

It is vital to note several caveats that come with this kind of classification. First, the geological units in Tanaka et al. [2014] are mapped at a coarse scale when compared to the size of impact craters in this study, because the authors primarily used MOLA ( 450 to $115 \mathrm{~m} / \mathrm{px})$ and THEMIS $(\sim 100 \mathrm{~m} / \mathrm{px})$ basemaps. Second, the supersets of geological units as defined in Table 2 are identified on the basis of features visible at the surface. It is important to bear in mind that the shapes of impact craters may be influenced by materials that underlie those exposed at the surface, and which have different mechanical properties. For example, lavas can overlie sediments and vice versa. The Vastitas Borealis unit $(\mathrm{lHl})$ has here been classified with "fine sediments" because it appears to contain sediment fill eroded from the highlands, but other features imply that lavas are intercalated with and underlie these sediments (Tanaka et al. [2014]). For this reason, and because Vastitas Borealis is so geographically extensive, we have assigned it to its own category. This does not imply, however, that other units are not also to some extent horizontally and vertically heterogeneous. The "purest" crater subpopulations, in the sense of forming in materials with relatively uniform material properties, are likely to be those in "lavas" and the "impact unit".

To this we add two geographical classifications: "highlands" versus "lowlands", based entirely on latitude and elevation, as well as "high latitude" and "low latitude", which are distinguished by latitudes exceeding or less than $45^{\circ}$, respectively. The "lowlands" subpopulation overlaps significantly with the set of craters that formed in Vastitas Borealis. High vs. low latitude categories are meant to identify regions with the most significant abundance of shallow subsurface ice (e.g., Boynton et al. [2002], Byrne et al. [2009]). We also identify the subpopulation of craters with lobate distal margins, suggesting a fluidized, ground-hugging flow. In the terminology proposed by Barlow et al. [2000], craters in the "lobate" subpopulation have layered ejecta, with a pancake or rampart terminus that exhibits some degree of sinuosity. Finally, to verify whether the distribution of measured quantities is in fact different for large and small craters, we define four additional categories comprising craters with diameters larger than $500 \mathrm{~m}$ and $1 \mathrm{~km}$, as well as smaller than $500 \mathrm{~m}$ and $1 \mathrm{~km}$.

We use the two-sided Kolmogorov-Smirnov test (hereafter "KS test") to compare the distributions of measured morphometric quantities (Press et al. [1988]). In particular, the KS test supplies a confidence level $(p)$ with which the KS statistic is likely to equal or exceed the value computed when comparing two populations that sample the same distribution. Put another way, $1-p$ is the probability with which we may reject the null hypothesis that two subpopulations have sampled the same distribution. Provided $p$ is small, we may be reasonably confident that two subpopulations exhibit different distributions. The KS test may be used in this way provided that the number of samples $n_{A}$ in set $A$ and the number of samples $n_{B}$ in set $B$ satisfy the relation $n_{e} \equiv n_{A} n_{B} /\left(n_{A}+n_{B}\right)>4$ (Press et al. $\left.[1988]\right)$. We note that the KS test is highly sensitive to differences in the median value, and not very sensitive to differences in the overall 
Table 4. Table of subpopulation comparisons: rim span and rim slopes. See also the caption to Table 3 for details.

\begin{tabular}{|c|c|c|c|c|c|c|c|c|}
\hline$q$ & Filter & A & B & $\left(n_{A}, n_{B}\right)$ & $q_{A} \pm \sigma_{A}$ & $q_{B} \pm \sigma_{B}$ & $p$ & ref. ID \\
\hline \multirow[t]{5}{*}{$\bar{\theta}_{1}^{\prime}$} & none & least modified & all others & $(63,303)$ & $0.29 \pm 0.11$ & $0.43 \pm 0.15$ & $<10^{-4}$ & $\mathrm{~m} 5$ \\
\hline & none & moderately modif. & highly modified & $(246,120)$ & $0.36 \pm 0.13$ & $0.50 \pm 0.16$ & $<10^{-4}$ & $\mathrm{~m} 6$ \\
\hline & none & modif. vis. L1 & modif. vis. L3 & $(127,40)$ & $0.39 \pm 0.16$ & $0.45 \pm 0.12$ & 0.0150 & $\mathrm{~m} 7$ \\
\hline & MM & high latitude & low latitude & $(39,207)$ & $0.40 \pm 0.16$ & $0.36 \pm 0.12$ & 0.0049 & i5 \\
\hline & MM & pure coarse & elsewhere & $(11,235)$ & $0.42 \pm 0.07$ & $0.36 \pm 0.13$ & 0.0200 & s7 \\
\hline \multirow[t]{5}{*}{$\theta_{2}^{\prime}$} & none & least modified & all others & $(67,311)$ & $0.49 \pm 0.11$ & $0.58 \pm 0.13$ & $<10^{-4}$ & $\mathrm{~m} 8$ \\
\hline & none & moderately modif. & highly modified & $(255,123)$ & $0.54 \pm 0.12$ & $0.62 \pm 0.13$ & $<10^{-4}$ & $\mathrm{~m} 9$ \\
\hline & MM & coarse deposits & elsewhere & $(18,237)$ & $0.59 \pm 0.09$ & $0.53 \pm 0.12$ & 0.0066 & s8 \\
\hline & MM & pure coarse & elsewhere & $(11,244)$ & $0.63 \pm 0.08$ & $0.53 \pm 0.12$ & 0.0011 & s9 \\
\hline & MM & impact unit & elsewhere & $(54,201)$ & $0.55 \pm 0.10$ & $0.53 \pm 0.13$ & 0.0330 & s10 \\
\hline \multirow[t]{4}{*}{$\phi_{c 1}$} & MM & large $(D \geq 1 \mathrm{~km})$ & small $(D<1 \mathrm{~km})$ & $(83,163)$ & $32.2 \pm 4.32$ & $23.2 \pm 6.24$ & $<10^{-4}$ & r1 \\
\hline & MM & large $(D \geq 500 \mathrm{~m})$ & $\operatorname{small}(D<500 \mathrm{~m})$ & $(100,146)$ & $31.6 \pm 4.96$ & $22.6 \pm 5.82$ & $<10^{-4}$ & r2 \\
\hline & MV1 & large $(D \geq 1 \mathrm{~km})$ & small $(D<1 \mathrm{~km})$ & $(44,83)$ & $33.7 \pm 3.08$ & $20.4 \pm 7.74$ & $<10^{-4}$ & r3 \\
\hline & MV1 & large $(D \geq 500 \mathrm{~m})$ & small $(D<500 \mathrm{~m})$ & $(56,71)$ & $31.5 \pm 7.02$ & $19.9 \pm 7.06$ & $<10^{-4}$ & $\mathrm{r} 4$ \\
\hline \multirow[t]{4}{*}{$\phi_{c 1}^{\prime}$} & none & least modified & all others & $(63,303)$ & $0.72 \pm 0.13$ & $0.59 \pm 0.16$ & $<10^{-4}$ & $\mathrm{~m} 10$ \\
\hline & none & moderately modif. & highly modified & $(246,120)$ & $0.66 \pm 0.13$ & $0.52 \pm 0.18$ & $<10^{-4}$ & $\mathrm{~m} 11$ \\
\hline & MM & pure coarse & elsewhere & $(11,235)$ & $0.63 \pm 0.05$ & $0.66 \pm 0.14$ & 0.0462 & s11 \\
\hline & MM & high latitude & low latitude & $(39,207)$ & $0.59 \pm 0.14$ & $0.67 \pm 0.13$ & 0.0019 & i6 \\
\hline \multirow[t]{4}{*}{$\phi_{c 2}$} & MM & large $(D \geq 1 \mathrm{~km})$ & small $(D<1 \mathrm{~km})$ & $(84,171)$ & $31.2 \pm 5.52$ & $15.0 \pm 6.82$ & $<10^{-4}$ & r5 \\
\hline & MM & large $(D \geq 500 \mathrm{~m})$ & small $(D<500 \mathrm{~m})$ & $(102,153)$ & $29.4 \pm 7.24$ & $14.3 \pm 6.26$ & $<10^{-4}$ & r6 \\
\hline & MV1 & large $(D \geq 1 \mathrm{~km})$ & small $(D<1 \mathrm{~km})$ & $(46,89)$ & $32.0 \pm 5.50$ & $12.4 \pm 6.49$ & $<10^{-4}$ & $\mathrm{r} 7$ \\
\hline & MV1 & large $(D \geq 500 \mathrm{~m})$ & $\operatorname{small}(D<500 \mathrm{~m})$ & $(59,76)$ & $28.2 \pm 9.36$ & $12.0 \pm 6.23$ & $<10^{-4}$ & r8 \\
\hline \multirow[t]{6}{*}{$\phi_{c 2}^{\prime}$} & none & least modified & all others & $(67,311)$ & $0.53 \pm 0.13$ & $0.45 \pm 0.14$ & $<10^{-4}$ & $\mathrm{~m} 12$ \\
\hline & none & moderately modif. & highly modified & $(255,123)$ & $0.49 \pm 0.13$ & $0.41 \pm 0.15$ & $<10^{-4}$ & $\mathrm{~m} 13$ \\
\hline & MM & coarse deposits & elsewhere & $(18,237)$ & $0.45 \pm 0.10$ & $0.49 \pm 0.14$ & 0.0104 & s12 \\
\hline & MM & pure coarse & elsewhere & $(11,244)$ & $0.40 \pm 0.08$ & $0.49 \pm 0.13$ & 0.0082 & s13 \\
\hline & MM & impact unit & elsewhere & $(54,201)$ & $0.47 \pm 0.11$ & $0.49 \pm 0.14$ & 0.0177 & s14 \\
\hline & MM & impact unit & lavas & $(54,61)$ & $0.47 \pm 0.11$ & $0.51 \pm 0.14$ & 0.0163 & s15 \\
\hline \multirow[t]{7}{*}{$\phi_{f}^{\prime}$} & none & least modified & all others & $(70,314)$ & $0.62 \pm 0.08$ & $0.53 \pm 0.12$ & $<10^{-4}$ & $\mathrm{~m} 14$ \\
\hline & none & moderately modif. & highly modified & $(261,123)$ & $0.57 \pm 0.12$ & $0.50 \pm 0.11$ & $<10^{-4}$ & $\mathrm{~m} 15$ \\
\hline & none & modif. vis. L1 & modif. vis. L3 & $(139,41)$ & $0.56 \pm 0.11$ & $0.53 \pm 0.07$ & 0.0421 & $\mathrm{~m} 16$ \\
\hline & MM & coarse deposits & elsewhere & $(18,243)$ & $0.50 \pm 0.09$ & $0.57 \pm 0.12$ & 0.0333 & s16 \\
\hline & MM & pure coarse & elsewhere & $(11,250)$ & $0.48 \pm 0.10$ & $0.57 \pm 0.12$ & 0.0085 & s17 \\
\hline & MM & high latitude & low latitude & $(42,219)$ & $0.60 \pm 0.17$ & $0.56 \pm 0.10$ & 0.0149 & i7 \\
\hline & MM & lobate ejecta & not lobate & $(32,229)$ & $0.62 \pm 0.19$ & $0.56 \pm 0.10$ & 0.0059 & i8 \\
\hline
\end{tabular}

shape of the distributions being compared. We therefore acknowledge it is possible there are some significant differences that we cannot identify using this test.

A list of all subpopulation comparisons for which $n_{e}>4$ and $p<0.05$ is supplied in Table 3 for quantities pertaining to cavity shape and dimensions, in Table 4 for the rim span and rim slopes, and in Table 5 for quantities pertaining to crater rim shape and dimensions. The column titled "Filter" contains any subset used to "filter" the database before making a comparison. For example, if subpopulation A is "highlands" and subpopulation B is "lowlands" and the filter is "MV1", then the comparison is between craters in MV1 that occur in the highlands and craters in MV1 that occur in the lowlands. This allows us to compare only craters that are not heavily modified, or indeed to examine the effects of modification if no filter is applied. In the following sections we will refer to these tables as we discuss details of the measured morphometric distributions.

Because some quantities are strong functions of diameter (see Section 5), before comparing subpopulations we have sought to remove this diameter dependence by subtracting fitted linear or power-law relationships where appropriate. These quantities were then normalized and rescaled so that they range from 0 to 1 . Failing to do this could reveal statistically significant differences between subpopulations that are largely the result of a diameter-dependent sampling bias. For example, comparatively fresh impact craters are more easily visible in THEMIS imagery and hence targeted for HiRISE observations when they occur in some terrains when compared with others (Tornabene et al. [2006]). Also, large craters may be preferentially well-preserved at high latitudes where small craters are swiftly erased by ice-related processes.

In Tables 3 through Table 5, these normalized and rescaled quantities are represented using a primed symbol. These are: the upper and lower rim spans $\left(\theta_{1}^{\prime}, \theta_{2}^{\prime}\right)$, middle and upper cavity wall slopes $\left(\phi_{c 1}^{\prime}, \phi_{c 2}^{\prime}\right)$ and flank slope $\left(\phi_{f}^{\prime}\right)$, as well as the two measures of upper cavity wall shape $\left(\xi_{c}^{\prime}, \ell_{c}^{\prime}\right)$. Because the diameter dependence is in some cases nontrivial and difficult to subtract (especially $\xi_{c}^{\prime}$ and $\ell_{c}^{\prime}$ ), we discard subpopulation comparisons in which the primed quantities exhibit a statistically significant difference between craters having $D>500 \mathrm{~m}$ and $D \leq 500 \mathrm{~m}$. Later, we show that $\alpha_{c}$ exhibits a nontrivial diameter dependence that is difficult to remove. We report results for $\alpha_{c}$ with the important caveat that diameter-dependent sampling may account for the differences that relate to geologic setting. 
Table 5. Table of subpopulation comparisons: rim shape and dimensions. See also the caption to Table 3 for details.

\begin{tabular}{|c|c|c|c|c|c|c|c|c|}
\hline$q$ & Filter & A & B & $\left(n_{A}, n_{B}\right)$ & $q_{A} \pm \sigma_{A}$ & $q_{B} \pm \sigma_{B}$ & $p$ & ref. ID \\
\hline \multirow[t]{3}{*}{$\overline{h / D}$} & MM & impact unit & elsewhere & $(49,201)$ & $0.03 \pm 0.01$ & $0.04 \pm 0.01$ & 0.0109 & s18 \\
\hline & MM & impact unit & lavas & $(49,61)$ & $0.03 \pm 0.01$ & $0.04 \pm 0.01$ & 0.0194 & s19 \\
\hline & MV1 & lobate ejecta & not lobate & $(14,120)$ & $0.04 \pm 0.01$ & $0.03 \pm 0.01$ & 0.0080 & i9 \\
\hline \multirow[t]{4}{*}{$\xi_{c}^{\prime}$} & none & least modified & all others & $(66,295)$ & $0.32 \pm 0.16$ & $0.40 \pm 0.15$ & $<10^{-4}$ & $\mathrm{~m} 17$ \\
\hline & none & moderately modif. & highly modified & $(244,117)$ & $0.37 \pm 0.17$ & $0.43 \pm 0.13$ & $<10^{-4}$ & $\mathrm{~m} 18$ \\
\hline & low lat. & moderately modified & highly modified & $(205,102)$ & $0.35 \pm 0.15$ & $0.41 \pm 0.12$ & 0.0001 & m19 \\
\hline & LM & lobate ejecta & not lobate & $(11,55)$ & $0.42 \pm 0.21$ & $0.30 \pm 0.14$ & 0.0303 & $\mathrm{i} 10$ \\
\hline \multirow[t]{12}{*}{$\ell_{c}^{\prime}$} & none & least modified & all others & $(65,310)$ & $0.49 \pm 0.15$ & $0.46 \pm 0.15$ & 0.0121 & $\mathrm{~m} 20$ \\
\hline & none & moderately modif. & highly modified & $(253,122)$ & $0.48 \pm 0.15$ & $0.45 \pm 0.16$ & 0.0141 & $\mathrm{~m} 21$ \\
\hline & none & modif. vis. L1 & modif. vis. L2 & $(133,202)$ & $0.51 \pm 0.16$ & $0.45 \pm 0.15$ & 0.0004 & $\mathrm{~m} 22$ \\
\hline & none & modif. vis. L1 & modif. vis. L3 & $(133,40)$ & $0.51 \pm 0.16$ & $0.44 \pm 0.11$ & 0.0339 & $\mathrm{~m} 23$ \\
\hline & low lat. & modif. vis. L1 & modif. vis. L2 & $(115,165)$ & $0.50 \pm 0.14$ & $0.45 \pm 0.13$ & 0.0016 & $\mathrm{~m} 24$ \\
\hline & low lat. & moderately modif. & highly modified & $(212,107)$ & $0.49 \pm 0.14$ & $0.43 \pm 0.13$ & 0.0017 & $\mathrm{~m} 25$ \\
\hline & MM & pure coarse & elsewhere & $(11,242)$ & $0.57 \pm 0.09$ & $0.47 \pm 0.15$ & 0.0259 & s20 \\
\hline & MM & fine deposits & elsewhere & $(47,206)$ & $0.42 \pm 0.16$ & $0.49 \pm 0.14$ & 0.0266 & i11 \\
\hline & MM & Vastitas Bor. & elsewhere & $(32,221)$ & $0.41 \pm 0.17$ & $0.49 \pm 0.14$ & 0.0051 & i12 \\
\hline & MM & high latitude & low latitude & $(41,212)$ & $0.43 \pm 0.19$ & $0.49 \pm 0.14$ & 0.0082 & i13 \\
\hline & MM & lobate ejecta & not lobate & $(31,222)$ & $0.41 \pm 0.19$ & $0.49 \pm 0.14$ & 0.0013 & i14 \\
\hline & MV1 & lobate ejecta & not lobate & $(13,120)$ & $0.43 \pm 0.15$ & $0.52 \pm 0.15$ & 0.0329 & i15 \\
\hline
\end{tabular}

\section{Measured morphometric distributions}

The quantities defined in Table 1 are plotted in Figures 5 through 7 and Figures 10 through 12. The majority of these plots illustrate how these quantities vary with crater diameter. Each plot displays results for the entire crater database, or for one or more subpopulations defined in Section 4 and Table 2. The number of craters represented in each plot, the meaning of the error bars, along with additional criteria used to filter the database, are reported in the figure captions.

\subsection{Crater cavity}

Early studies using MOLA track profiles and/or gridded topography to characterize the scaling of crater shapes on Mars focused largely on $D>1 \mathrm{~km}$ (e.g., Garvin and Frawley [1998], Garvin et al. [2003]). Later work has suggested that these data sets can be used to produce robust measurements of quantities such as $d / D$ or $h / D$ with tolerable uncertainties for $D>6 \mathrm{~km}$ (Stewart and Valiant [2006]) or only for $D>10 \mathrm{~km}$ (Robbins and Hynek [2013]). Because along-track spacing of MOLA is $\sim 300 \mathrm{~m}$ and cross-track spacing reaches $1.5 \mathrm{~km}$ near the equator (Smith et al. [2001]), a strong aliasing effect causes crater depth and rim height to be

under-estimated for simple craters (Robbins and Hynek [2013]; i.e., this happens when altimeter measurements miss the deepest part of the crater floor or the tallest part of the crater rim).

Several studies have measured scaling relationships for important morphometric quantities including rimto-floor depth versus rim diameter, and some of these are plotted in Figure 5 along with our measurements. When comparing these results to ours, it is important to bear in mind two caveats. First, it should be emphasized that the scaling laws derived from other studies are relevant to a prescribed range of diameters significantly exceeding those examined in our study.

Second, we note that crater depth has been measured differently in many studies. In the present work, we measure the distance between average rim height (after subtracting the rim-fitted plane) and the lowest elevation of the crater floor. The other studies to which we compare our results also measure rim-to-floor depths. Stepinski et al. [2009] measured "the difference between the highest and the lowest elevation in a depression" from gridded MOLA topography. Robbins and Hynek [2012a] used gridded MOLA topography to find the difference between average rim height and the lowest floor elevation, consistent with the approach taken by Boyce et al. [2005, 2006] and Boyce and Garbeil [2007]. Robbins and Hynek [2013] demonstrated the importance 
of using non-interpolated MOLA elevations in estimating the rim-to-floor depth, as measured from the mean of above-average rim heights to the mean of belowaverage floor depths. Stewart and Valiant [2006] measured depth from crater rim to crater floor (to obtain the scaling laws we show here), where the rim height was derived from individual MOLA tracks and the cavity floor elevation was measured from gridded MOLA topography. Garvin et al. [2000] and Garvin et al. [2003] measured the maximum rim height along individual MOLA tracks that cross the crater center (see Figure 2 in Garvin et al. [2000]).

These prior studies have characterized the dependence of rim-to-floor depth upon diameter using a power law, $d=c D^{-m}$ for constants $c$ and $m$, usually in the range $\mathrm{D}>5 \mathrm{~km}$. Here we supply our own estimate of this relationship, using the "deepest craters" method described in Boyce and Garbeil [2007] and Robbins and Hynek [2012a]. That is, a power law was fit to the deepest craters in a set of diameter bins. We use 15 diameter bins of equal size in $\log (D)$ that span the range of craters in our study from $D>50 \mathrm{~m}$ (i.e., excluding the few smallest craters, most of which clearly depart from the trend; see Figure 5). The results of fitting a line to the $1,2,3,4$, and 5 deepest craters in each bin were highly consistent: $c$ ranges from 0.22 to 0.21 , while $m$ is within $1 \%$ of 1.01 in all cases. Fitting to the five deepest craters in each bin, we find (for $N=75$ craters and $d$ and $D$ in meters):

$$
d=(0.205 \pm 0.012) D^{1.012 \pm 0.009}
$$

We also note that fitting to craters with $50 \mathrm{~m} \leq D \leq 500$ $m$ yields a somewhat larger exponent of $m=1.05 \pm 0.03$. Like all other scaling laws presented in this paper, we have reported this result for $D$ in meters because we have focused on the range of small crater sizes (largely $D<1 \mathrm{~km}$ ). The $d$ versus $D$ relationship is usually reported in $\mathrm{km}$, however, and we supply this result for comparison:

$$
d=(0.223 \pm 0.013) D^{1.012 \pm 0.009}
$$

for $d$ and $D$ in $\mathrm{km}$ (i.e., $c$ in equation (1) has been multiplied by $\left.10^{3(m-1)}\right)$. In what follows, please note that values of $c$ cited from prior literature are for $d$ and $D$ in $\mathrm{km}$. The trend for the deepest craters plotted in Figure 5 is most consistent with the early photogrammetric and shadow-length measurements of R. J. Pike (summarized in Pike [1980b]), who found that $m \approx 1$ and $c \approx 0.2$ for fresh impact craters on Mars. This result $(d \sim 0.2 D)$ is similar to what he measured for fresh simple craters on the Moon (Pike [1974]) as well as Mercury (Pike [1988]).

The majority of studies that have estimated $c$ and $m$ for $D>1 \mathrm{~km}$ using MOLA and, more recently, HRSC-derived DEMs (Robbins and Hynek [2013]) have found $m<1$ (with the exception of Stepinski et al. [2009] who found $m>1.5$, and Robbins and Hynek [2012a], who found $m \approx 1$ but later revised their re- sult based on updated data and analysis (Robbins and Hynek [2013])). As such, when we extrapolate these previously-published relationships to small sizes, they tend to overestimate the depths that we have measured. The majority of scaling laws in Stewart and Valiant [2006] overestimate depths for $D<1 \mathrm{~km}$, while Garvin et al. [2003] and Robbins and Hynek [2013] are consistent with the deepest craters in our population except for $D<150 \mathrm{~m}$. It has been noted before that early MOLA-derived scaling laws implied extremely deep craters $(d / D>0.3)$ at small diameters (McEwen et al. [2005]).

For now, we offer several possible explanations for this discrepancy between our result and the extrapolated scaling laws for larger craters: (1) we have so far measured too few craters: with more measurements, uncommonly deep craters will populate the space between our results and the large-diameter scaling laws; (2) small impact craters on Mars are modified so quickly that craters in this size range are rapidly "shallowed" to depths below the scaling laws; (3) our sample population at small sizes is dominated by distant secondaries

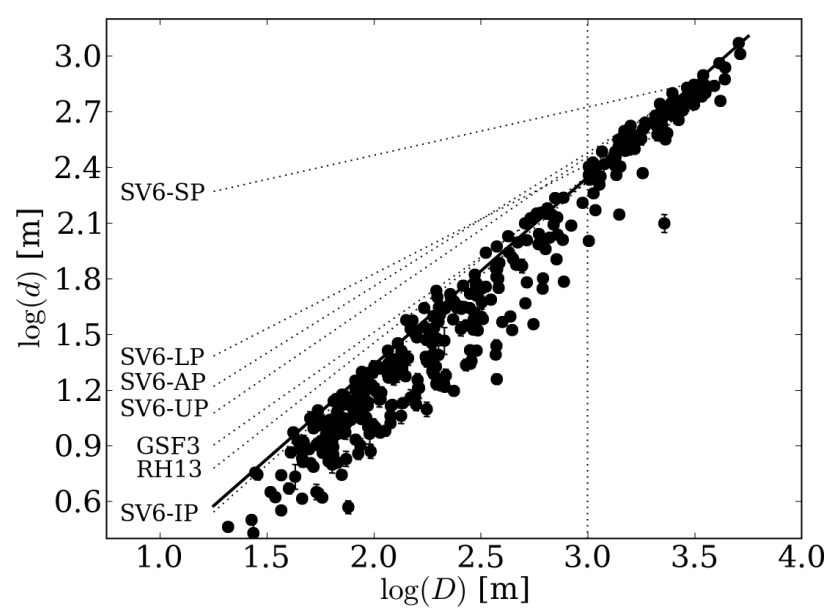

Figure 5. Average rim-to-floor depth $(d)$ versus diameter $(D)$ from the present study (filled circles). Also plotted are scaling laws $d=c D^{-m}$ measured for large simple craters on Mars, here extrapolated to the small diameter range. In what follows, $c$ is reported for $d$ and $D$ in kilometers. From Stewart and Valiant [2006] (SV6, for AP $=$ Acidalia Planitia: $(c, m)=(0.302,0.720)$, $\mathrm{UP}=$ Utopia Planitia: $(c, m)=(0.288,0.790), \mathrm{IP}=\mathrm{Isidis}$ Planitia: $(c, m)=(0.213,1.02), \mathrm{LP}=$ Lunae Planum: $(c, m)=(0.261,0.590), \mathrm{SP}=$ Solis Planum: $(c, m)=$ $(0.532,0.260))$ for $D>3 \mathrm{~km}$; Garvin et al. [2003] (GSF3: $(c, m)=(0.21,0.81)$, similar to $(c, m)=(0.25,0.82)$ as found in Boyce et al. [2006]); and Robbins and Hynek [2013] (RH13: $(c, m)=(0.227,0.901))$ for $D>2 \mathrm{~km}$ from HRSC DEMs. The global estimate of Pike [1980b], $(c, m)=(0.204,1.019)$ plots close to SV6-IP, which is nearest to our result. We fitted a line to the 5 deepest craters in 15 diameter bins (solid black line) to obtain $(c, m)=(0.223 \pm 0.013,1.012 \pm 0.009)$. Error bars represent standard deviation of depth, equal to standard deviation of rim height, and are commonly smaller than symbol size. (Plot: $N=384$; Fit: $N=75$ ) 
that excavate to shallower depths; (4) coarse-sampling and aliasing effects in MOLA DEMs for $D<10 \mathrm{~km}$ have led to errors in MOLA-derived scaling laws; (5) large simple craters are subject to gravity-driven failure of crater walls that increase with cavity size (e.g., Pike [1988]), so that a different scaling law governs this regime; (6) atmospheric drag tends to slow smaller projectiles so that small craters form via proportionately less-efficient excavation. We cannot completely rule out (1) - (3) but (4) seems unlikely in light of the careful consideration of uncertainties in Stewart and Valiant [2006] and Robbins and Hynek [2012a], and noting once more that their results are not, in general, inconsistent with ours for large diameters. Robbins and Hynek [2013] point out that aliasing leads to underestimating the depth of small craters, which causes the $d$ vs. $D$ relation to become more shallow rather than steeper. The remaining possibilities (5) and (6) will be examined more closely in Section 6.3.

Simple impact crater cavities on Mars have long been known to resemble a cone, paraboloid, or intermediate shape (e.g., Garvin and Frawley [1998]). We measure the cavity shape by means of a nonlinear leastsquares fit of a power law $\left(z=B r^{\alpha_{c}}\right)$ to radial profiles of the cavity and report the exponent, $\alpha_{c}$. A perfectly paraboloid crater will have $\alpha_{c}=2$ while a perfectly conical crater will have $\alpha_{c}=1$. The dependence of $\alpha_{c}$ on diameter is plotted in Figure 6 for all craters except those with a major step or bench in the cavity walls (such as caused by craters forming in layered

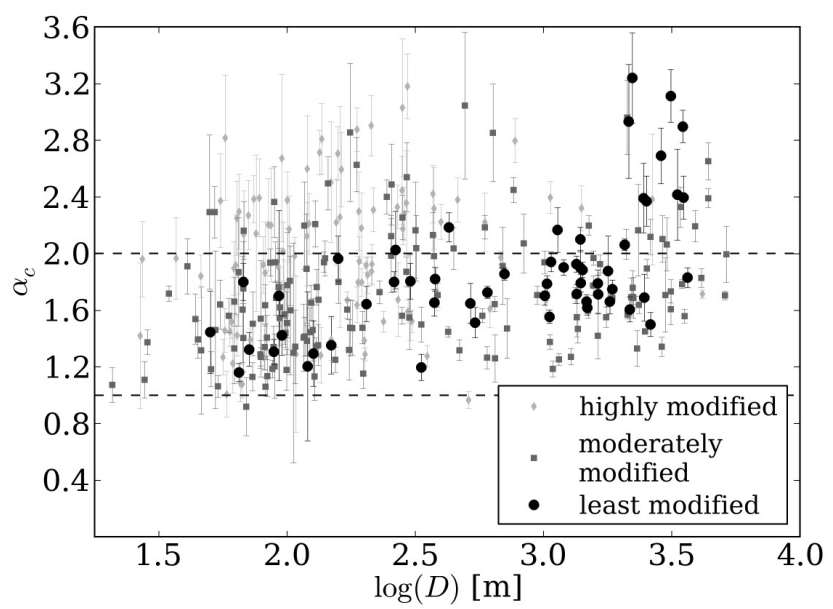

Figure 6. Dependence of cavity shape exponent $\alpha_{c}$ on diameter. Small craters $(D<500 \mathrm{~m})$ tend to be more conical (nearer $\alpha_{c}=1$ ) than large craters, which are more parabolic in cross-section (nearer $\alpha_{c}=2$; see also Table 3, a1-a6). As craters are modified and filled, this exponent also increases on average (see also Table 3, m1-m2). Even extremely fresh craters exhibit $\alpha_{c}>2$ for $D>2.5 \mathrm{~km}$ as might be expected from failure of cavity walls nearing the simple-complex transition. $(N=367$; craters in MM (includes LM) and HM, omitting craters with prominent benches or steps in cavity walls that span $>180^{\circ}$. Error bars represent uncertainty of the azimuthal mean values.)

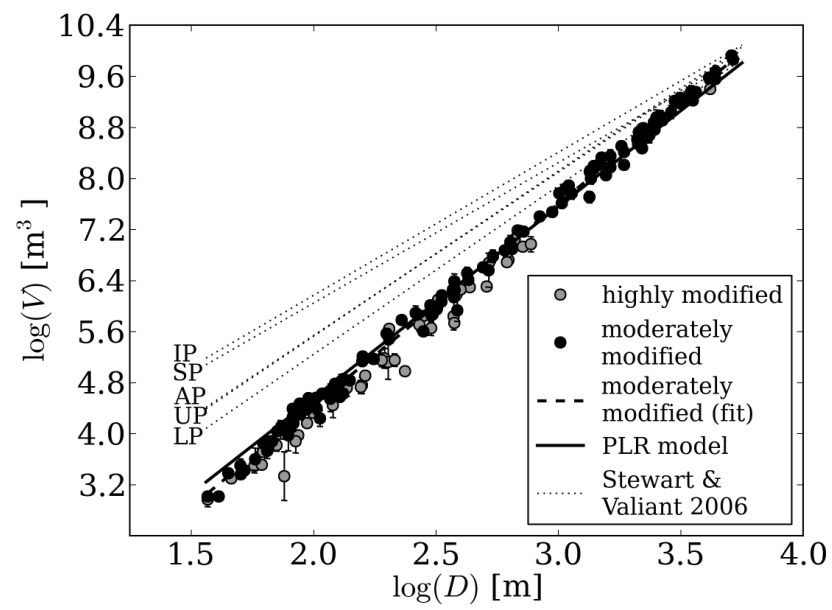

Figure 7. Cavity volume versus crater rim diameter. Power-law fit to craters in subpopulation MM is given by equation (3), shown by the dashed line. PLR refers to the "power law of revolution" in equation (4). Also plotted are scaling laws reported in Stewart and Valiant [2006] for below-surface cavity volume versus surface diameter of large simple craters $(D>3 \mathrm{~km})$, here extrapolated to small crater sizes. See text for discussion, and caption to Figure 5 for region codes. (Error bars represent the uncertainties described in Section 3.2. Fit includes $N=128$ craters in MM; excluded are elevation models with $>10 \%$ interpolated elevations in the cavity. Plot includes HM craters (gray dots), for $N=180$ in total.)

rock of varying strength) that spans more than $180^{\circ}$ (more than $50 \%$ of the intermediate "circumference"). The fit is made to the cavity wall from B to E in Figure 3: we omitted the distance $0.1 R$ from the center (to avoid the influence of minor sediment fill) and $0.1 R$ nearest to the rim (to avoid the influence of rim curvature). We find a mean value of $\alpha_{c}=1.75 \pm 0.12$ with a standard deviation of 0.43 for moderately modified craters (MM). When fitting to the entire radial profile, we find $\alpha_{c}=1.66 \pm 0.11$ with a standard deviation of 0.37 (also for MM craters). Elsewhere in this paper, we have reported only the measurement that makes use of $80 \%$ of the profile (from B to $\mathrm{E}$ in Figure 3 ). In Table 3, however, we have reported statistically significant differences in $\alpha_{c}$ between subpopulations only if this difference is statistically significant for both measurements.

Our result is in good agreement with the average exponent $\alpha_{c}=1.75$ (from MOLA profiles) and $\alpha_{c}=1.68$ (from gridded MOLA) measured by Garvin et al. [2003] for large simple craters (diameter range not reported, but likely $D>1 \mathrm{~km}$ as in Garvin and Frawley [1998]). This suggests that crater cavity shape is not a strong function of diameter below the simple-complex transition at $D \approx 5 \mathrm{~km}$. However, we find a statistically robust tendency for a more conical shape to be favored at small sizes among relatively unmodified craters (especially $D<500 \mathrm{~m}$; see Table 3, a1-a6). Super-parabolic shapes $\left(\alpha_{c}>2.0\right)$ occur more often at larger sizes and 
in modified craters (see Figure 6 and Table 3, m1-m2). Whereas $43 \%$ of craters with $D<500 \mathrm{~m}$ have $\alpha_{c}<1.5$, this is true of only $14 \%$ of craters with $D>500 \mathrm{~m}$. A significant fraction of the least modified (LM) craters have $\alpha_{c}>2.0$ for $D>2.5 \mathrm{~km}$, owing to flatter floors that may have resulted from sediment infilling or partial collapse of the upper cavity walls for diameters approaching the simple-complex transition. An expanded discussion of these results is saved for Section 6.3.

As mentioned, we have also measured the diameter dependence of crater volume, plotted in Figure 7. In this case, we have confined our estimates to DEMs in which $<10 \%$ of the cavity has interpolated elevations and only craters belonging to MM. We find the relation (with standard uncertainties from a least-squares linear fit, for $N=128)$ :

$$
V=(0.0129 \pm 0.0012) D^{(3.16 \pm 0.015)},
$$

for $D$ in meters and $V$ in $\mathrm{m}^{3}$. Also in Figure 7, we have plotted the volume for a "power law of revolution" (PLR): i.e., the rotation of a cavity cross-section defined by the power law $z^{*}=B r^{* \alpha_{c}}$, where $r^{*} \equiv r / R$ is the normalized radius, $z^{*} \equiv z / R$ is the radius-normalized elevation, and $D=2 R$. Note that if $d=0.2 D$, then $B=0.4$. Integrating $z^{*}=B r^{* \alpha_{c}}$ from $r^{*}=0$ to $r^{*}=1$, the volume of the PLR is given by:

$$
V=\frac{B \pi D^{3}}{16\left(2 / \alpha_{c}+1\right)}
$$

which is in good agreement with our measurements when $\alpha_{c}=1.75$, the mean cavity exponent measured earlier (see black solid line in Figure 7). That is, the diameter dependence of crater volume for the craters in our database $(D<5 \mathrm{~km})$ is well-approximated by the PLR with constant aspect ratio $d / D=0.2$ and exponent 1.75. The PLR model slightly underestimates the volume at large diameters, and slightly overestimates at small diameters, likely owing to the diameterdependence of $\alpha_{c}$ discussed earlier (i.e., larger $\alpha_{c}$ for higher $D$ would slightly boost the volume estimate, while smaller $\alpha_{c}$ for smaller $D$ would slightly reduce it.)

Stewart and Valiant [2006] computed the diameter dependence of cavity volume (below elevation of surrounding surface) as a function of surface diameter (diameter measured at the surrounding surface elevation) in five regions on Mars for simple craters with $D>6$ $\mathrm{km}$, fitting a relation $V \sim D^{p}$ and finding that $p<3$, consistent with $m<1$ in the relation $d \sim D^{-m}$. These scaling relationships are also plotted in Figure 7 , where they have been extrapolated to the small crater size range, and significantly overestimate the volumes we measured for $D<1 \mathrm{~km}$. Note that since equation (3) and Stewart and Valiant [2006] approximately agree for
$D \sim 6 \mathrm{~km}$, their result $p<3$ implies that volumes at small sizes will be overestimated.

It should be emphasized that Stewart and Valiant [2006] measured the volume below the elevation of the surrounding surface as a function of surface diameter, whereas our crater volumes extend somewhat above this surface (see Section 3.2) and are plotted as a function of rim diameter. We have compared with their results because Stewart and Valiant [2006] remarks that the cavity volume measured up to the rim "shows the same trend" in diameter. We conclude that small simple craters on Mars exhibit a diameter dependence that is relatively straightforward when compared with their larger counterparts in the simple-complex transition regime, where increased gravity-driven failure of crater walls complicates this dependence.

\subsection{Crater rims}

Many studies have used qualitative assessments of the "sharpness" or "crispness" of crater rims to assist with identifying fresh craters (e.g., Wood and Anderson [1978], Garvin et al. [2000], McEwen et al. [2005]). This appears justified since crater rims are softened and rounded by some surface processes such as soil and ice creep (e.g., Squyres and Carr [1986]) and because they are lowered and widened by wind erosion (e.g., Grant et al. [2006]). In a photogrammetric study, Craddock et al. [1997] found that complex crater rims on Mars are rounded on geologic time scales. Whitehead et al. [2010] found that complex craters with sharp rims tend also to be less filled with sediments. In this section we examine the dependence of rim shape and "rim span" upon diameter, through new quantitative estimates of the "roundness" of rims and the slopes of the cavity and flank surfaces.

Two simple craters from our database are shown in Figure 8. Figure $8 \mathrm{~A}$ shows a small crater $(D=50 \mathrm{~m})$ formed in the past decade whose rim does not appear "sharp" at the resolution of this HiRISE image. The crater in part $(\mathrm{B})$ of this figure is much older and larger $(D \approx 1400 \mathrm{~m}$ ) with an apparently "sharp" rim when viewed at this scale. Closer inspection of the rim in (C) reveals mass-wasting features: ridges alternating with downslope debris chutes reminiscent of landslides. Examples of azimuthally-averaged radial profiles of crater rims, scaled by crater radius $\left(r^{*} \equiv r / R, z^{*} \equiv z / R\right)$, are plotted in Figure 9, alongside estimates of each crater's diameter. Figures 8 and 9 illustrate cases where the scaled rim shape of larger craters is "sharper" (less rounded) than smaller craters. Bearing in mind this informal and qualitative observation (sharper rims at larger diameters), we turn now to examine the morphometric diameter dependence of rim shape for a large population of relatively unmodified craters.

As mentioned earlier, rim "roundness" has been estimated using two length scales: a characteristic length scale of radial changes in slope ("rim decay length") on the cavity wall $\left(\ell_{c}\right)$ and flank $\left(\ell_{f}\right)$ and the radius-of- 

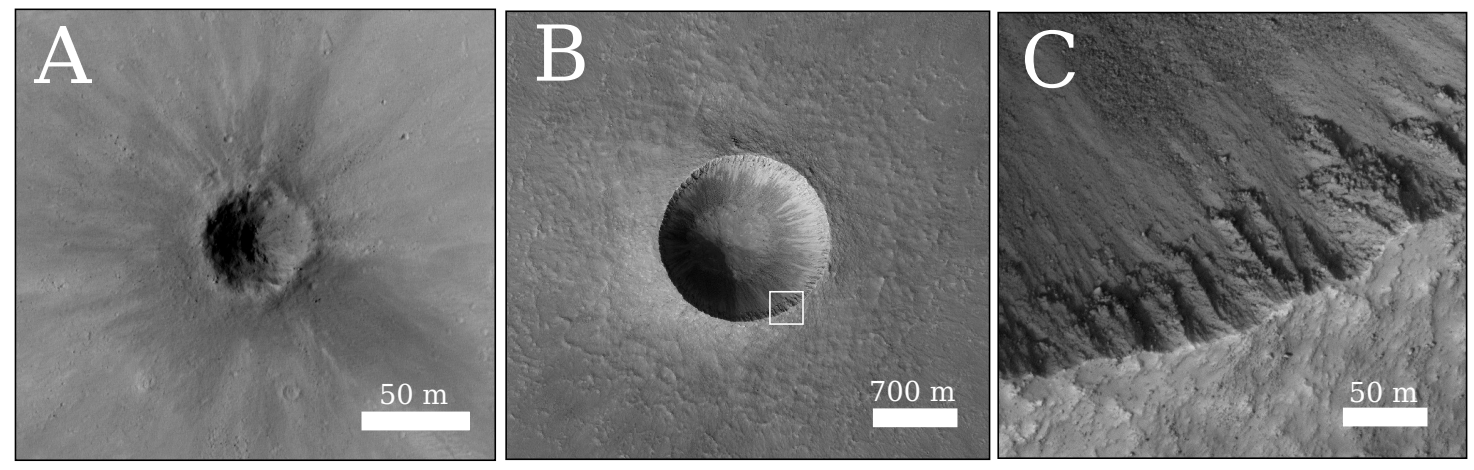

Figure 8. (A) Simple crater that formed between 2003 and 2009, from HiRISE image ESP_011425_1775 with $D \approx 50 \mathrm{~km}$ and "rounded" rim. (This crater does not appear in THEMIS observation I06419047 from 2003.) The averaged radial profile for this crater is shown at the bottom of Figure 9. (B) Wellpreserved simple bowl-shaped crater in HiRISE image ESP_012857_1910. (C) Magnified view of crater rim in (B) with ridge-and-chute morphology ("R\&C").

curvature near the rim on the cavity wall $\left(\xi_{c}\right)$ and flank $\left(\xi_{f}\right)$. These measures of rim "roundness" are plotted versus crater diameter in Figures 10 and 11. In the former case, the rim decay length on the upper cavity wall increases with crater diameter, approximately following a power-law (for $\ell_{c}$ and $D$ in meters and $D<1 \mathrm{~km}$ ):

$$
\ell_{c}=(0.124 \pm 0.015) D^{(0.838 \pm 0.026)} .
$$

The scatter in $\ell_{c}$ increases significantly at $D \approx 1 \mathrm{~km}$ as this length scale shortens (the rim sharpens) for many craters. We find a similar trend and transition in the plot of the radius-of-curvature for the upper cavity wall ( $\xi_{c}$; see Figure 11 ). At $D \approx 1 \mathrm{~km}$ this quantity starts to deviate from the fitted power-law trend (fitted for $D<1 \mathrm{~km})$ :

$$
\xi_{c}=(0.329 \pm 0.045) D^{(0.851 \pm 0.027)}
$$

for $\xi_{c}$ and $D$ in meters. That the radius-of-curvature increases for many craters with $D>1 \mathrm{~km}$ suggests that the upper cavity walls are flattened (rims sharpened) in an azimuthally-averaged sense. That is, it should be emphasized that these quantities represent the averaged result for the entire crater. That a transition appears to occur at $D \approx 1 \mathrm{~km}$ does not imply that only craters larger than $D=1 \mathrm{~km}$ will exhibit a flat surface in some part of the upper cavity walls (a partly sharpened rim). Instead, this indicates that many craters larger than $D=1 \mathrm{~km}$ exhibit sharp rims (flat upper cavity walls) around most or all of their circumference. As the radius-of-curvature approaches infinity, the fit uncertainty also grows very large, so that individual measurements of a large radius-of-curvature contribute little to the weighted average that is plotted on these graphs. These figures also show that craters with $D>1$ $\mathrm{km}$ are dominated by mass-wasting features (ridge-andchute morphology) as seen in Figure 8C.

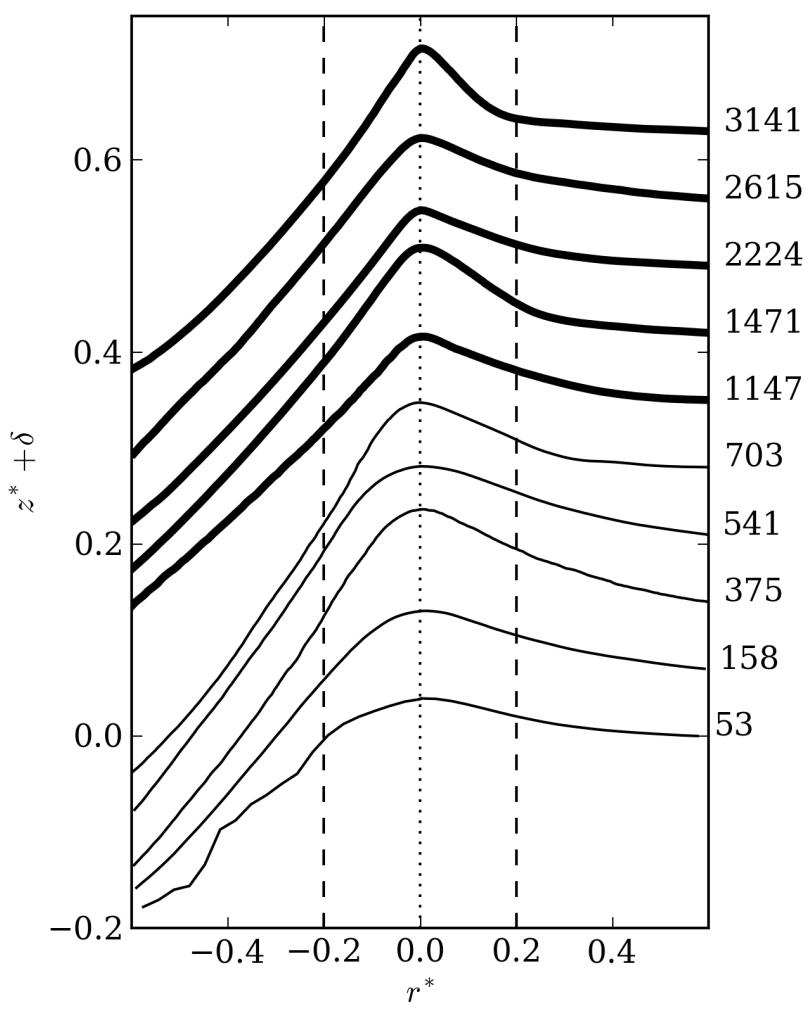

Figure 9. Azimuthally-averaged radial profiles for five craters with $D>1 \mathrm{~km}$ (bold lines) and five craters with $D<1 \mathrm{~km}$ (thin lines). Crater diameter in meters is listed at the right, and the axes represent radius-normalized elevation and radial position $\left(z^{*} \equiv z / R, r^{*} \equiv r / R\right.$, for crater radius $R$ ). The bottom-most profile was measured from the fresh impact crater shown in Figure 8A. Craters with $D>1 \mathrm{~km}$ exhibit a flatter upper cavity wall and narrower rim span (a sharper rim) on average, consistent with trends and transitions observed in the diameterdependence of rim shape parameters shown in Figures 10 through 14. 


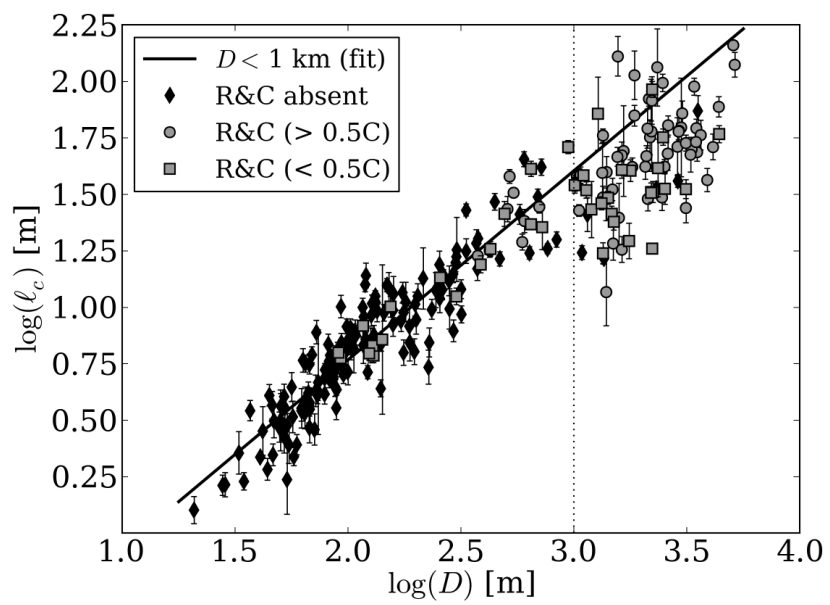

Figure 10. Rim decay length measured on the upper cavity walls $\left(\ell_{c}\right)$ versus crater diameter. This length scale exhibits a roughly power-law trend in diameter until $D \approx 1 \mathrm{~km}$, when a significant fraction of crater rims are "sharpened" (the upper cavity wall is flattened). Craters in this size range are dominated by the ridge-and-chute (R\&C) morphology typical of landsliding shown in Figure $8 \mathrm{C}$. Circles indicate craters in which the mass-wasting features are noted around more than $50 \%$ of the crater circumference ("> $0.5 \mathrm{C}$ "), while squares indicate they are present around $<50 \%$ of the circumference. (Fit: $N=169(D<1 \mathrm{~km}, \mathrm{MM}) ;$ Plot: $N=261(\mathrm{MM}))$

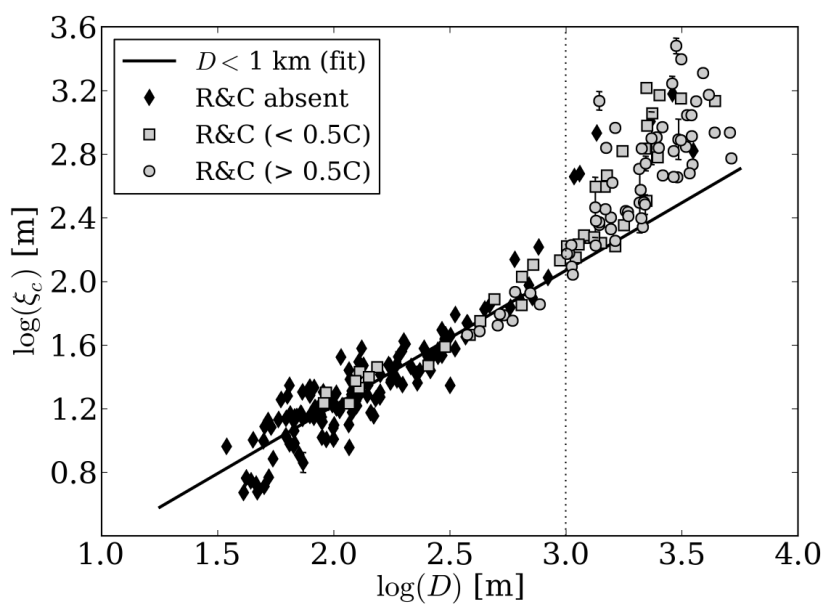

Figure 11. Radius-of-curvature of upper cavity wall $\left(\xi_{c}\right)$ versus crater diameter. Radius-of-curvature appears to follow a power-law trend until $D \approx 1 \mathrm{~km}$. Above this diameter, the upper cavity walls appear to flatten (i.e., the rims sharpen) along most of the rim circumference in many craters. See caption to Figure 10 for symbol definitions. The standard uncertainties are smaller than the markers in most cases. (Fit: $N=161(D<1 \mathrm{~km}, \mathrm{MM})$; Plot: $N=261(\mathrm{MM}))$

Power-law fits for the corresponding measures of "roundness" on crater flanks are reported here but not plotted, and do not exhibit an equally marked transition at $D \approx 1 \mathrm{~km}$ :

$\ell_{f}=(0.211 \pm 0.013) D^{(0.684 \pm 0.011)}$, $\xi_{f}=(0.273 \pm 0.032) D^{(1.008 \pm 0.019)}$,

for $\ell_{f}, \xi_{f}$, and $D$ in meters $(N=161$ and $N=169$, respectively). The absence of a transition at $D \approx 1 \mathrm{~km}$ is discussed later in Section 6.3. We have also measured the dependence of rim height on diameter. In good agreement with prior studies (Pike [1977], Fudali et al. [1980], Robbins and Hynek [2012b], Stewart and Valiant [2006]), the average rim height is a few percent of diameter. For MM craters, we consider only those with sufficient surrounding topography to estimate the baseline elevation on a per-profile basis, and exclude craters perched on local topographic maxima $(N=250)$. We find (for $h$ and $D$ in meters):

$$
h=(0.0354 \pm 0.0028) D^{(1.017 \pm 0.013)} .
$$

Although we have not plotted the data used to derive this result, it should be noted that we detect no transition in the slope of $\log (h)$ vs. $\log (D)$ : that is, we observe no transition in the diameter dependence of rim height for $D<5 \mathrm{~km}$. A fraction of the high-latitude craters exhibit a thin layer of ejecta that extends beyond the limits of the DEM. For this reason, the $h$ vs. $D$ relation that we measure may slightly underestimate the true rim height for a few of the largest craters in this study.

Finally, we measured three slopes in the vicinity of the crater rim, in addition to the rim span (the angle subtended by the cavity-facing and flank-facing rim slopes). The "middle cavity slope" $\left(\phi_{c 1}\right)$ is measured by fitting a line between positions $\mathrm{C}$ and $\mathrm{E}$ (see Figure 3 ) and the "upper cavity slope" $\left(\phi_{c 2}\right)$ is measured by fitting a line between $\mathrm{D}$ and $\mathrm{F}$ in Figure 3. Although the former is called "middle" to distinguish it from "upper", it is measured high on the cavity wall but avoids most of the curvature of the upper cavity wall nearest

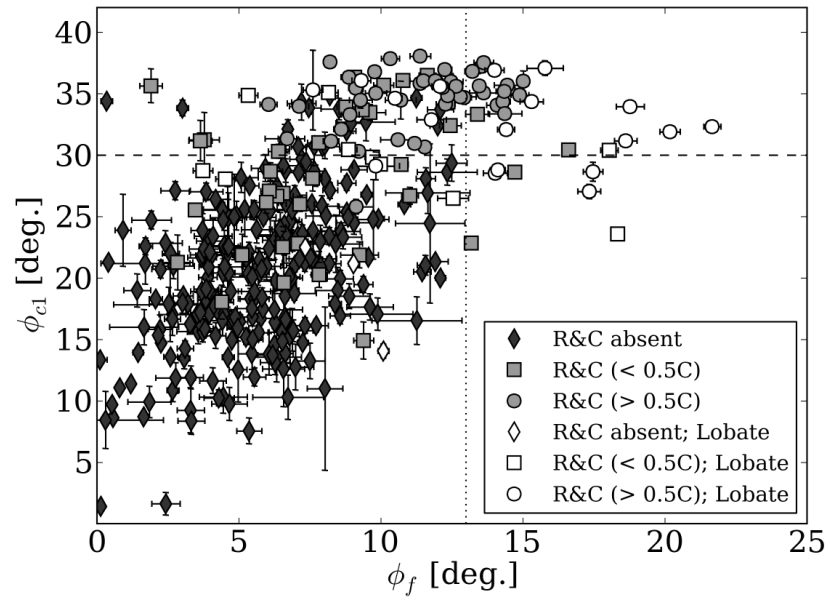

Figure 12. Middle cavity wall slope versus flank slope. Crater rims with $\phi_{c 1}>30^{\circ}$ dominantly exhibit ridge-andchute morphology $(\mathrm{R} \& \mathrm{C})$ as shown in Figure 8C. Fifty percent of craters with $\phi_{f}>13^{\circ}$ exhibit lobate ejecta (open markers). See caption to Figure 10 for symbol definitions. Error bars represent uncertainty of azimuthallyaveraged values. $(N=364)$ 

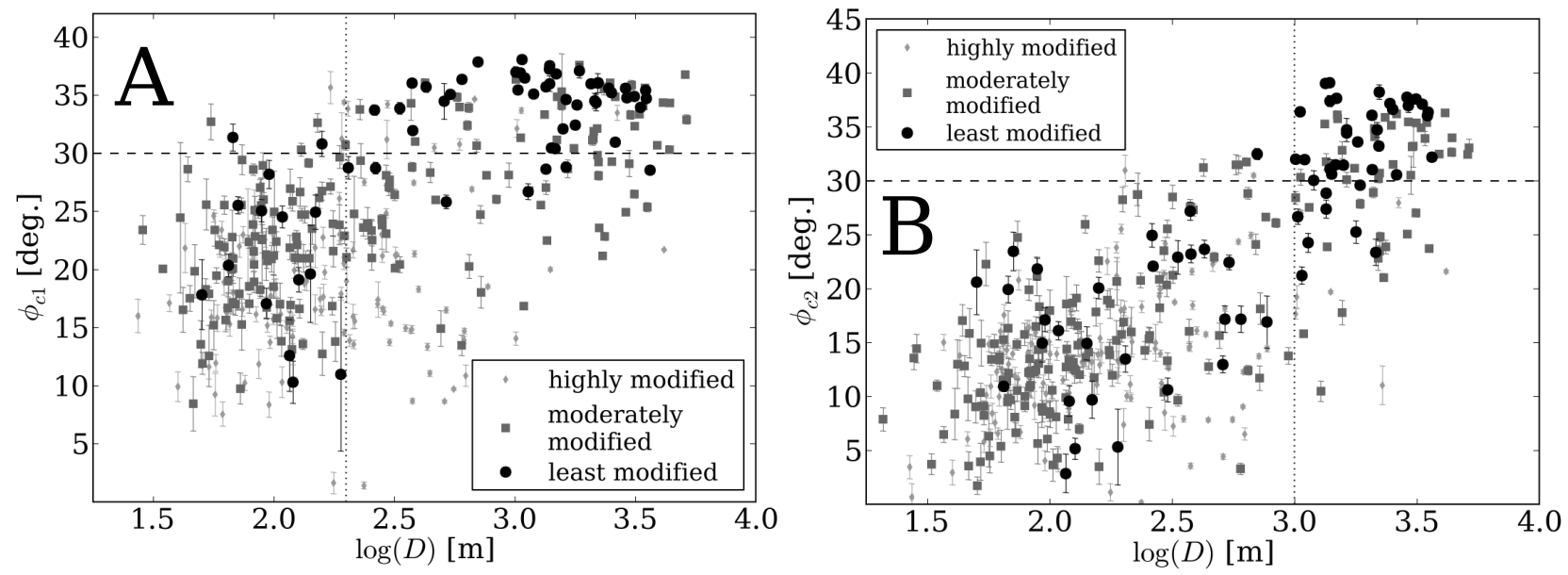

Figure 13. (A) Middle cavity wall slope versus crater diameter. Slopes typically exceed $30^{\circ}$ for $D>250$ $\mathrm{m}$ and are usually smaller than this value for $D<250 \mathrm{~m}$. $(N=377$ for craters in MM (includes LM) and HM.) (B) Upper cavity wall slope versus crater diameter. Slopes usually exceed $30^{\circ}$ for $D>1 \mathrm{~km}$ and are usually lower than this value for $D<1 \mathrm{~km}$. Error bars represent the uncertainty of azimuthally-averaged values. $(N=366$, for craters in MM (includes LM) and HM)
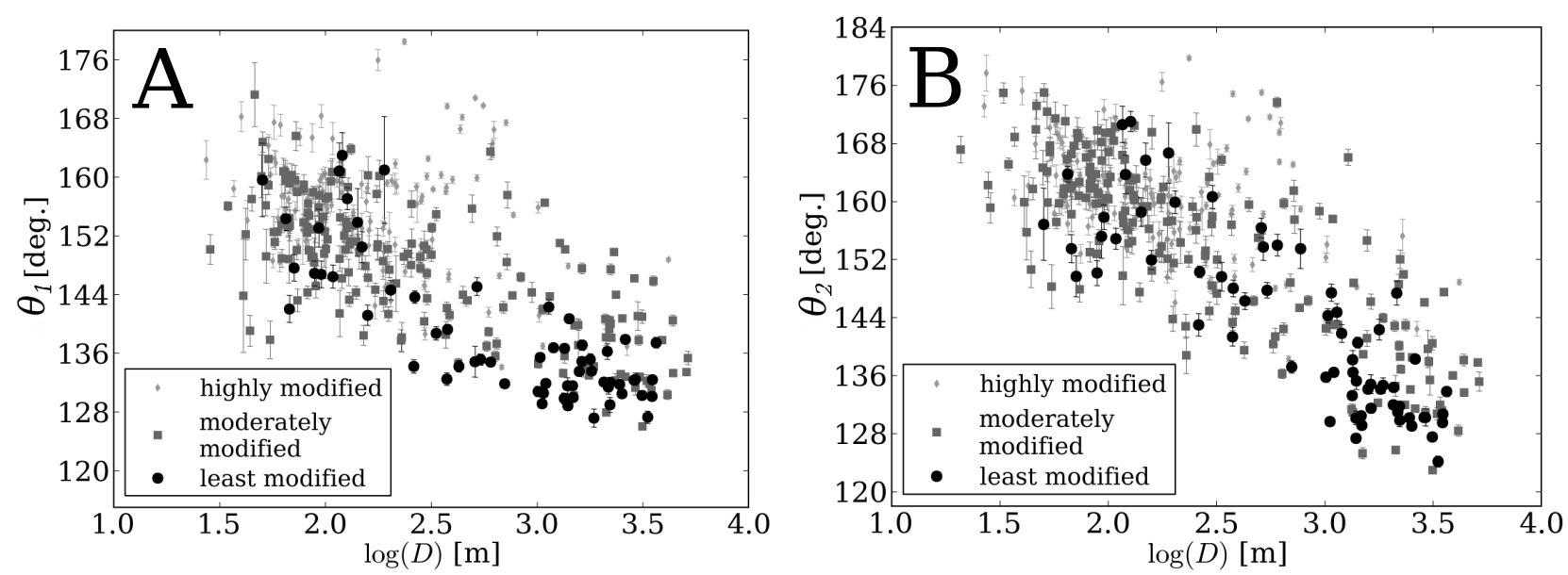

Figure 14. (A) Lower rim span versus crater diameter. Broadly, rim span narrows as diameter increases and widens as craters are modified $(N=364$, for craters in HM and MM (includes LM)). (B) This trend is also apparent in the upper rim span $\left(\theta_{2}\right)$, which exhibits a strong negative correlation with $\log D$ (Pearson coefficient $\rho=-0.75$ for MM and $\rho=-0.79$ for LM). The diameter dependence of $\theta_{2}$ is described by equation (10) in the text. Error bars represent the uncertainty of azimuthally-averaged values. (Plot shows $N=375$, for craters in HM and MM (includes LM)).

the rim. Middle cavity slopes are typical of the steepest slopes in the cavity walls. The dependence of both quantities on diameter is shown in Figure 13. We also measured the flank slope $\left(\phi_{f}\right)$ using a linear fit from position $\mathrm{F}$ to $\mathrm{G}$, whose dependence on diameter is not plotted.

The angle of repose is approximately equal to the angle of internal friction; it is independent of planetary gravity and usually exceeds $30^{\circ}$ for planetary surface materials (Carson and Kirkby [1972]). Pike [1977] found that cavity slopes reach a maximum of about $29^{\circ}$ at the simple-complex transition for lunar craters. We find that the azimuthally-averaged middle cavity slope $\left(\phi_{c 1}\right)$ is dominantly above $30^{\circ}$ for $D>250 \mathrm{~km}$ and usu- ally below $30^{\circ}$ for $D<250 \mathrm{~km}$, as shown in Figure $13 \mathrm{~A}$. By contrast, the upper cavity slope $\left(\phi_{c 2}\right)$ usually exceeds $30^{\circ}$ only for $D>1 \mathrm{~km}$ as seen in 13B, approximately coincident with the transitions observed in Figures 10 and 11 for rim decay length and curvature radius. That the distributions of these slopes are statistically distinct for the small and large diameter regime is confirmed by KS test comparisons in Table 4, r1-r8.

From the rim and flank slopes we compute the upper and lower "rim span" (see Figure 3, Table 1): the angle subtended by the cavity-facing and flank-facing rim slopes, defined as: $\theta_{1} \equiv 180^{\circ}-\left(\phi_{c 1}+\phi_{f}\right)$ and $\theta_{2} \equiv 180^{\circ}-\left(\phi_{c 2}+\phi_{f}\right)$. Smaller rim spans (especially of $\theta_{2}$ ) imply a "narrower" or "steep-sided" rim. The dependence of rim span on diameter is shown in Figure 
14. It is expected to vary between $180^{\circ}$ (flat rim) and about $120^{\circ}$ : the span implied by the angle of repose on the cavity- and flank-facing slopes of the rim. The upper rim span exhibits a strong negative correlation with log diameter ( $\rho=-0.79$ for LM craters), where this dependence can be approximated by:

$$
\theta_{2} \approx\left(200^{\circ} \pm 4^{\circ}\right)-\left(20^{\circ} \pm 2^{\circ}\right) \log D
$$

for LM craters $(N=67)$ and $D$ in meters. This relation anticipates that the "critical" (or over-steepened) rim span $\theta_{2} \approx 120^{\circ}$ is reached at $D \approx 10 \mathrm{~km}$ on average, in excess of the simple-complex transition diameter on Mars (Robbins and Hynek [2012a]).

We have also plotted the middle cavity slope versus the flank slope in Figure 12. This indicates that craters with middle cavity slopes exceeding $30^{\circ}$ are dominated by mass-wasting features, as expected. Kreslavsky and Head [2006] found that a threshold slope of $\sim$ $20^{\circ}$ distinguished craters with similar features (from those without) at high latitudes $\left(>52^{\circ}\right)$. Figure 12 shows that such features occur in our global population throughout the range $\phi_{c 1}=20^{\circ}$ to $\phi_{c 1}=30^{\circ}$ in a substantial minority of craters.

Finally, the regime of steep upper cavity slopes and flank slopes (upper right corner of Figure 12) is dominated by craters with lobate ejecta. An example profile from such a crater is plotted at the top of Figure 9. This profile exhibits a marked break in slope on the distal flanks, and has the smallest rim span. Roughly half of craters with $\phi_{f}>13^{\circ}$ and nearly all craters with $\phi_{f}>15^{\circ}$ exhibit lobate ejecta. We consider this result more closely in Section 6.3.

\section{Discussion}

In this section we provide an expanded discussion of the results, including implications for understanding the short-term modification of impact craters on Mars, the dependence of shape characteristics on geologic setting, as well as the diameter-dependent transitions we have observed in cavity and rim shape.

\subsection{Short-term modification}

Before discussing the implications of our results for the short-term modification of the craters in our study, it should be emphasized that we have sampled a global population. That is, a morphometric analysis of regionspecific populations are likely to exhibit trends and relationships that are not captured here. Also, this discussion concerns "short-term" modification because we have examined the relationship between modification and morphometry for a population of relatively wellpreserved craters.

As mentioned already in Section 4, the diameternormalized rim-height $h / D$ is significantly correlated with the normalized rim-to-floor depth $d / D$ only for craters that appear least modified (MV1; see Figure 4). With increasing modification, this correlation dimin- ishes, which implies that the erosion of rims and the filling of cavities is often decoupled. The comparison of relatively modified and unmodified subpopulations reveals a statistically significant difference for $d / D$ as expected, where $d / D$ decreases with increasing modification (Table 3, m3-m4). Surprisingly, we do not find a statistically-significant difference in $h / D$ between MV1, MV2, and MV3. That is, the distribution of diameternormalized rim height in this global population is not significantly different for craters that exhibit visual hallmarks of modification to varying degrees.

We find a tendency for $\alpha_{c}$ to increase as craters become increasingly modified (see Table 3, m1-m2 and Figure 6). That is, cavities become more paraboloidal or super-paraboloidal $\left(\alpha_{c}>2\right)$ as modification increases. This is consistent with filling and flattening of crater floors and/or steepening of crater walls, although we consider the former to be more likely except where mass-wasting dominates. Overall, we find a weak negative correlation between $\alpha_{c}$ and diameter-normalized rim-to-floor depth $(d / D)$ : Pearson's correlation coefficient $\rho=-0.35$ for $N=311$ craters lacking major topographic steps in their cavity walls. This is consistent with the notion that increasing modification (decreasing $d / D)$ tends to increase $\alpha_{c}$, on average.

The slope-related parameters that we have measured for rim shape exhibit expected trends with modification. Rim spans widen as modification increases (see Table 4, m5-m9) as a result of the concomitant decrease in rim and flank slopes (see Table 4, m10-m16).

Linear diffusion models have long been used to describe modification of impact craters whose surface evolution is dominated chiefly by soil creep (e.g., Soderblom [1970], Fassett and Thomson [2014]) and have been applied to understanding the modification sequence of complex craters on Mars (e.g., Craddock et al. [1997]). If the starting condition is a paraboloidal crater with a sharp rim (flat upper cavity wall), topographic diffusion will drive the rim toward a more rounded shape over time. This is also a consequence of creep in ice-rich regolith, believed to account for the "terrain softening" observed at mid- to high-latitudes on Mars (Squyres and Carr [1986], Jankowski and Squyres [1993]). Rounding of rims has also been observed for the long-term degradation of martian complex craters in highland terrains (Craddock et al. [1997]).

By contrast, for our global population of small, relatively well-preserved simple craters, we find a statistically significant tendency for upper cavity walls to flatten rather than become more rounded, with increasing modification (Table 5, m17-m18, m20-m23). That is, on average, the curvature radius of the upper cavity wall is larger and cavity rim decay length is shorter for craters that appear to be more modified.

Fully $85 \%$ of our globally-sampled crater population is dominated by low latitude craters (|lat. $\mid<45^{\circ}$, see Table 2). Since the dominant surface processes are known to be different for ice-rich versus ice-poor ma- 
terials, we repeated the comparison for only low latitude craters, finding that upper cavity walls are indeed flatter, on average, for craters that are more modified (see Table 5, m19, m24-m25). By contrast, repeating the same analysis for high-latitude craters ( $\mid$ lat. $\mid \geq 45^{\circ}$ ) we find no statistically significant difference for $\xi_{c}$ or $\ell_{c}$ between the relatively modified and unmodified subpopulations. This may suggest that, on average, advective processes like mass wasting dominate the shortterm modification at low latitudes, in contrast to the ice-related creep that has been observed by others to govern the surface evolution at high latitudes.

Although we find a flattening of upper cavity walls in low latitudes, it should be emphasized that this observation applies to the short-term modification of craters because we have examined a relatively well-preserved population. Moreover, this observation applies to the rim morphometry in an azimuthally-averaged sense. It is well-known from casual inspection of radial profiles in our study that modified crater rims exhibit a variation in shape, and that the dominant shape may also change from region to region. We expect that different processes likely dominate the long-term modification of crater rims. For example, the upper cavity wall may flatten in response to mass-wasting, known to dominate the early modification (Grant [1999]), and later become more rounded as diffusive processes take on an increasingly important role.

\subsection{Terrain dependence}

Many prior studies have shown that $d / D$ tends to be larger for fresh impact craters forming in stronger materials (e.g., Pike [1980b], Boyce et al. [2006], Stewart and Valiant [2006], Robbins and Hynek [2012a], Whitehead et al. [2010]), because stronger cavity walls are likely to support steeper slopes. In this section we will discuss the dependence of morphometric parameters on geologic setting, largely as a function of strength, and only for relatively unmodified craters (MM and MV1). The subpopulations of impact craters that formed in specific terrains are defined in Section 4 and Table 2, and are based largely on the map of Tanaka et al. [2014]. In what follows, we report only on the statistically-significant differences found in the comparisons reported in Tables 3 through 5 .

The "lavas" population comprises all craters that formed in lava flows and flood lavas of Amazonian and Hesperian age, while the "volcanics" population includes pyroclastic and volcaniclastic flows in addition to lava flows. Of all the geologic subpopulations in Table 2, we assume that these materials are the strongest. Curiously, lavas and volcanics were flagged in the case of only three statistically-significant comparisons. Namely, $d / D$ is slightly larger for craters that formed in lavas and volcanics when compared with all other craters, or when compared with craters forming in the impact unit (see Table 3, s2-s4). Crater rims are also taller (larger $h / D$ ), on average, in lavas when compared with impact unit materials (see Table 5, s19).

The "impact unit" subpopulation refers to craters that formed inside of other craters or their ejecta. Most of these materials consist of displaced fragmented rock, and thus are considered a relatively weak target. Coarse-grained deposits ("coarse deposits") are also considered relatively weak for similar reasons. This subpopulation is based only on Tanaka et al. [2014] and overlaps with the "impact unit": "coarse deposits" refer to Tanaka et al. [2014]'s AHi impact unit, as well as units that contain drop moraines, mass wasting products, and catastrophic debris flow deposits. "Pure coarse deposits" are units thought to be dominated by these materials. We find that $d / D$ is on average somewhat smaller for craters in the "pure coarse deposits" and "impact unit" subpopulations (see Table 3, s5-s6). That $d / D$ is larger in stronger materials is consistent with the findings of other studies based on populations of larger craters on Mars (Boyce et al. [2006], Stewart and Valiant [2006], Whitehead et al. [2010], Robbins and Hynek [2012a]).

Unsurprisingly, we find that rim slopes are smaller, on average, in the weaker geologic targets (see Table 4, s11-s17), causing rim spans to be wider in the same settings (Table 4, s7-s10). There is little evidence to suggest a clear-cut relationship between rim shape (curvature and decay length) and target strength. We find only one statistically significant difference for the very small subpopulation of MM craters in pure coarse deposits $(N=11)$ : rims in these materials have larger $\ell_{c}$ (rounder upper rim walls), on average (Table 5, s20). There is likewise little evidence to suggest a clear-cut conclusion about how the cavity shape exponent $\alpha_{c}$ depends on terrain type. We find that $\alpha_{c}$ is significantly smaller in the impact unit: on average somewhat closer to conical $\left(\alpha_{c}=1\right)$ than others, which are closer to parabolic in cross-section $\left(\alpha_{c}=2\right.$; see Table $\left.3, \mathrm{~s} 1\right)$.

We have saved for last the difficult problem of understanding the influence of high-latitude and lowland terrains. This poses a challenge because the mechanical properties of the high latitudes and lowlands are not precisely known. We define lowlands as latitude $>0^{\circ}$ and elevation $<-3 \mathrm{~km}$ (as defined in the MOLAderived elevation model of Smith et al. [2001] and marked in Figure 1). The lowlands are likely a layered mixture of highland-derived sediments, impact regolith, and lava flows (Tanaka et al. [2014] and references therein). This overlaps significantly with the subpopulation we have identified as "fine sediments", which is primarily made up of the extensive Vastitas Borealis population (unit "lHl" in Tanaka et al. [2014]). As mentioned earlier, the mechanical properties of this terrain are not well understood. The "highlands" vs. "lowlands" comparison yields no statistically significant differences in morphometry among relatively unmodified craters (MM and MV1) according to the KS test. 
High-latitude terrains (defined as $\mid$ lat. $\mid>45^{\circ}$ in this study) are ice-rich in the shallow subsurface (e.g., Boynton et al. [2002], Byrne et al. [2009]), which is expected to weaken these materials and result in the formation of shallow cavities, shallow rims, and ice-filled crater floors (Senft and Stewart [2008]). Accordingly, we find that relatively well-preserved high-latitude craters exhibit some hallmarks of craters forming in weak target materials: smaller upper cavity slopes and broader rim spans (see Table 4, i5-i6). We identify no statistically significant difference in $d / D$ and $h / D$ between low and high latitudes for relatively unmodified craters (MV1, MM, LM).

Curiously, we also find some features of craters forming in ice-rich targets that seem not to be consistent with weak materials. For example, craters with lobate ejecta margins exhibit taller rims (larger $h / D$ ), deeper cavities (larger $d / D$ ) that are more bowl-shaped (larger $\alpha_{c}$ ), and steeper flank slopes (see Tables 3-5, i2, i4, i8i9). Lobate-ejecta craters, as well as high-latitude and Vastitas craters also exhibit sharper rims (flatter upper cavity walls; see Table 5, i10-i15). These observations are difficult to reconcile with expectations (Senft and Stewart [2008]) except by suggesting that the ice-rich layer is thin and underlain by stronger rocks, such as the flood lava plains thought to underlie the northern lowlands (Tanaka et al. [2014] and references therein). It is also important to point out that craters strongly affected by the mechanism described in Senft and Stewart [2008] are unlikely to be identified as belonging to MM or MV1 in the first place.

Complex craters with lobate ejecta and steep flank slopes have been noted by others (Boyce and MouginisMark [2006]), and have been explained in terms of the oversteepening and subsequent collapse of the rimproximal flank during excavation (Barnouin-Jha et al. [2005]). Our work shows that the large flank slopes of fresh lobate-ejecta simple craters are statistically anomalous. In our global sample, those with a flank slope $\phi_{f}>15^{\circ}$ usually exhibit lobate ejecta (see Figure 12). Lobate-ejecta craters also have a significantly larger flank slope on average (see Table 4, i8). Future work using high-resolution DEMs could usefully map the relationship between flank slope and ejecta mobility, ejecta aspect ratio, layering, and rampart morphometry, as part of ongoing efforts to understand the emplacement of layered ejecta (e.g., Weiss and Head [2014]).

\subsection{Diameter-dependent transitions}

As mentioned earlier, prior studies of large simple craters near the simple-complex transition diameter have found scaling laws for crater depth and volume that generally overestimate these quantities when extrapolated to the sizes of craters addressed here $(D<5$ $\mathrm{km})$. A possible explanation is that gravity-driven collapse of crater walls "begins" at very small diameters on Mars. As diameter increases, the first expression of sig- nificant gravity-driven modification may be the partial collapse and related "sharpening" of crater rims as discussed in Section 5.2. It may be for this reason that the power-law dependence of $d$ upon $D$ is steeper at smaller sizes than has been previously measured for larger diameters, as discussed earlier in Section 5.1.

Well-preserved impact crater rims appear to exhibit a somewhat rounded shape that is well-described by the power-law relations supplied in equations (5) through (8) for $D<1 \mathrm{~km}$. As mentioned, many crater rims become sharper above this diameter (upper cavity walls flatten, see Figures 10 and 11) and commonly display signs of mass-wasting (see Figure $8 \mathrm{C}$ ). Although the middle cavity wall commonly exceeds a slope of $30^{\circ}$ for many craters with $D<1 \mathrm{~km}$ (as shown in Figure 13A), the transition in rim shape that occurs at $D \approx 1 \mathrm{~km}$ is also expressed in the diameter-dependence of slopes on the upper cavity walls $\left(\phi_{c 2}\right)$. This dominantly exceeds $30^{\circ}$ only for $D>1 \mathrm{~km}$ (Figure 13B). That is, slopes characteristic of the repose angle normally do not reach the rim itself (along a major part of the rim circumference) except when $D>1 \mathrm{~km}$. Flank slopes $\left(\phi_{f}\right)$ are generally shallower by comparison, and typically do not reach the repose angle for any diameter. It may be for this reason that we do not observe an equally marked transition in flank curvature or decay length $\left(\xi_{f}\right.$ and $\ell_{f}$, respectively).

We cannot tell, however, if the transition at $D \approx 1$ $\mathrm{km}$ is a primary feature of the crater-forming process in this size range, or whether the scaling laws in equations (5) and (6) also hold for fresh craters in the range $D>1 \mathrm{~km}$, whose rims then sharpen over short time scales. If we plot $\xi_{c}$ vs. $D$ for all craters in our database (including highly modified craters), we find signs that the transition diameter shifts to a lower value $(D \approx 700 \mathrm{~m})$. As suggested by results in Section 6.1, crater rims may begin with a partially-rounded shape and then sharpen with the early stages of crater modification, which are dominated by mass wasting (Grant [1999]). As other processes become important, such as wind-driven erosion and soil creep, crater rims are further lowered and widened over time. As mentioned earlier, long-term modification may cause the rims to become more rounded again if diffusive processes govern the long-term evolution. In either case, these findings suggest that caution must be applied when using qualitative assessments of rim "sharpness" to identify relatively fresh craters in the range of small diameters $(D<1 \mathrm{~km})$.

Finally, we have shown that the cavity shape exponent $\left(\alpha_{c}\right)$ is closer to 1 , on average, for small craters (see Figure 6 and Table 3, a1-a6). We consider the hypothesis that this may reflect the Odessa vs. Barringer styles of crater formation associated with a difference in the effective penetration depth of the impactor (Shoemaker [1960], Shoemaker and Eggleton [1961], Shoemaker et al. [2005]). Relatively superficial explosions, caused by smaller projectiles that are more significantly 
slowed and disrupted by passage through the atmosphere, may result in Odessa-style craters that tend to be more conical in overall shape. By contrast, the more deeply-penetrating projectiles of higher-velocity impacts (and larger projectiles) more readily produce the bowl-shaped, excavation-dominated cavities akin to Barringer Meteor crater near Winslow, AZ, USA (Shoemaker et al. [2005]).

On Mars, this "transition" would be expected to occur at much smaller diameters than on Earth, owing to the much thinner atmosphere. From a very small sample size, a "transition diameter" for the Odessa vs. Barringer transition on Earth is ill-defined; one may estimate this to be $D \approx 200$ to $800 \mathrm{~m}$ based on seven classified craters: five in Shoemaker et al. [2005], in addition to Odessa and Barringer Craters (Shoemaker and Eggleton [1961]). If this accounts for the transition in $\alpha_{c}$, then the less efficient excavation of small, Odessatype craters on Mars may also explain a steeper $d$ vs. $D$ relationship at small diameters (because smaller craters would not be as deep, on average). Based on current models, however, objects of the size required to create craters $\sim 100 \mathrm{~m}$ in diameter, even if extremely weak and subject to disruption, are not expected to be significantly slowed by the very thin modern martian atmosphere (Popova et al. [2003]).

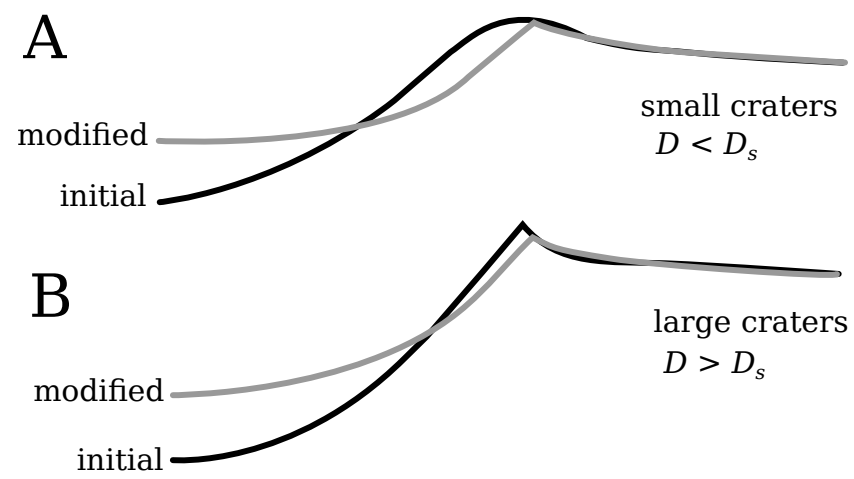

Figure 15. Initial shape and short-term modification of simple impact craters on Mars, as implied by the results in this study. (A) Cartoon illustrating common initial profile (black) and profile after short-term modification (gray) for small simple craters $\left(D<D_{s}\right.$ where $D_{s} \approx 1$ $\mathrm{km}$ ) in low-latitude environments likely dominated by initial mass-wasting, wind-mediated erosion, and infilling (rather than ice-related creep). The rim is initially somewhat rounded and cavity shape is closer to conical $\left(\alpha_{c}\right.$ closer to 1) than what occurs for larger craters on average. As the crater is modified by erosion and infilling, the cavity becomes super-paraboloidal $\left(\alpha_{c}>2\right)$. The upper cavity wall flattens $\left(\xi_{c} \uparrow, \ell_{c} \downarrow\right)$ as the rim retreats and the crater diameter and rim span increase. (B) Cartoon illustrating common initial profile (black) and profile after short-term modification (gray) for large simple impact craters on Mars. Initial profile is more paraboloidal $\left(\alpha_{c}\right.$ closer to 2) than smaller craters on average; initial angular span is narrower, rim slopes are steeper, and upper cavity wall is flatter than small craters. Modification increases $\alpha_{c}$ and the rim span.
We also cannot rule out that our sample population, in the range of small diameters, is dominated by distant secondaries that would have formed with a lower impact velocity, resulting in somewhat conical shapes. Alternatively, the observed diameter dependence of cavity shape could be ascribed to the faster modification of smaller craters, if it were not that $\alpha_{c}$ appears to increase, on average, with increasing modification, as discussed earlier in Section 6.1.

\section{Conclusions}

We have reported the dependence of morphometric characteristics upon diameter, terrain, and modification state, for a globally-distributed population of relatively well-preserved simple craters on Mars, as measured in high-resolution digital elevation models derived from stereo HiRISE imagery. We have extended the analysis of martian crater morphometry to smaller sizes than has been possible up to now $(25 \mathrm{~m}<D<5 \mathrm{~km})$. These results supply new insights about the relationships between morphometric parameters, and as such provide important constraints for models of landscape evolution and crater formation. In this section, we summarize some of the major observations described in this work, and refer readers to Section 6 for a deeper exploration of the significance of these results.

1. With increasing levels of modification, all of the following trends are observed on average: (a) cavity shape becomes more parabolic or super-parabolic $\left(\alpha_{c} \uparrow\right)$; (b) rim slopes become more shallow $\left(\phi_{c 1} \downarrow, \phi_{c 2} \downarrow\right.$ ,$\left.\phi_{f} \downarrow\right)$ and the rim span widens $\left(\theta_{1} \uparrow, \theta_{2} \uparrow\right)$; (c) for low-latitude craters ( $\mid$ lat. $\left.\mid<45^{\circ}\right)$, rims become sharper in the sense that upper cavity walls tend to flatten $\left(\xi_{c} \uparrow, \ell_{c} \downarrow\right)$. (See Figure 15.) On average, diameternormalized depth $(d / D)$ is smaller in the population of relatively modified craters, whereas the distribution of diameter-normalized rim height $(h / D)$ does not exhibit a statistically significant difference.

2. Excluding highly modified craters, cavity cross section is well-described by $z \sim r^{\alpha_{c}}$ for radial distance $r$, where $\alpha_{c} \approx 1.75 \pm 0.12$, in agreement with prior studies of larger craters. We also find a tendency for wellpreserved small craters to be more conical (smaller $\alpha_{c}$ ) than larger craters, which for $D>2.5 \mathrm{~km}$ are sometimes super-parabolic in cross-section $\left(\alpha_{c}>2\right)$ possibly on account of slumping.

3. Scaling laws from prior studies derived from MOLA and HRSC DEMs, when extrapolated to the size range of small craters $(D<5 \mathrm{~km})$, tend to overestimate the rim-to-floor depth and volume of simple craters in our database (especially for $D<150 \mathrm{~m}$ ). The volume of well-preserved craters scales approximately as $D^{3}$, as expected from a simple power-law-of-revolution model (PLR; equation (4)).

4. All aspects of rim shape exhibit a strong diameter dependence. Rim slopes tend to steepen with increas- 
ing diameter. The upper rim span decreases linearly with the log of diameter (see Figure 14) as described by equation (10). Two length scales that reflect the "roundness" of crater rims $\left(\xi_{c}, \ell_{c}\right)$ are well-described by power-law diameter scaling up to $D \approx 1 \mathrm{~km}$, above which the rims of many craters in our database sharpen significantly (Figures 10 and 11). Related to this transition, we also find that the slopes of upper cavity walls do not usually exceed typical repose angles $\left(\sim 30^{\circ}\right)$ except for $D>1 \mathrm{~km}$ (Figure 13B).

5. Craters that formed in strong materials (e.g., lavas) are deeper on average than craters that formed in weak materials (e.g., crater floors and ejecta), consistent with the results of previous studies. Rim slopes are more shallow in weaker materials as expected. Surprisingly, many well-preserved craters formed in ice-rich terrains (at high latitudes, or exhibiting lobate ejecta margins) exhibit features consistent with high-strength targets: steep flank slopes, taller rims, and deeper, bowlshaped cavities.

6. After simple post-processing steps described in Appendix B, elevation models generated using the Ames Stereo Pipeline 2.0 from HiRISE stereo image pairs agree with published DEMs generated using SOCET SET to within $\sim 0.5 \mathrm{~m}$ of elevation on average (after a translation to minimize the discrepancy).

We have not so far addressed the morphometry of crater rim planform shape, which is saved for later work. Later investigations will also explore the morphometry of secondary impact crater populations from Zunil and Gratteri craters, for which the present study of primaries will supply a useful basis for comparison.

A third important avenue for future study is to measure in detail the relationship between morphometric parameter values at the regional scale and in this way characterize the "modification sequence" of impact crater shapes. At the regional scale, we expect these parameters will not vary as much as we have measured in our global sample. These measurements will supply an important data set for comparing to the predictions of recent modeling efforts (e.g., Forsberg-Taylor et al. [2004]), especially in regard to surface evolution through the Amazonian epoch. The present study provides a more accurate initial condition for these models, and builds on the traditional suite of morphometric parameters for characterizing how crater shape evolves over time.

\section{Appendix A: Crater modification index}

There is currently no universally-agreed standard for quantifying the extent of crater modification. This is partly the result of variation in the way different surface processes are expressed in crater shape as well as the diverse goals of different analyses. The "crater modification index" introduced in this study, $i_{\mathrm{M}}$, is a single num- ber that is meant to represent the modification state of an impact crater based on visual inspection alone. It is defined as the sum of values that have been assigned to visually-assessed attributes. Attributes with positive values (that increase $i_{\mathrm{M}}$ ) are hallmarks of modification processes, such as a filled cavity or crater-pocked ejecta, while negative values suggest exceptionally good preservation. Because ejecta are the most transient feature of impact craters (e.g., Tornabene et al. [2006], Geissler et al. [2010]), the preservation of ejecta has been assigned the most weight (the largest range) in our scale. The apparent sharpness of crater rims has not been used because we sought to quantify this independently and hence avoid an a priori selection effect that could influence our results. All attributes were assessed by visual inspection of HiRISE and THEMIS night-IR imagery; these are defined and noted in the database published online with the supplementary materials for this article. The attributes and their associated values ("scores") are defined in Table 6. As discussed in Section 4, the positive correlation between scaled rim height $(h / D)$ and crater depth $(d / D)$ is largest for the craters with the smallest $i_{\mathrm{M}}$ (least modified), as expected.

\section{Appendix B: Digital elevation models}

In this appendix we describe how the elevation models used in this study were generated using the free and open source Ames Stereo Pipeline 2.0 (ASP) and in-house post-processing scripts. Then we discuss a comparison of 20 ASP models to 20 models of the same craters cropped from DEMs published by the HiRISE Science Team and generated using BAE Systems' SOCET SET. We find that the average per-pixel deviation of ASP-derived DEM elevations from the elevation in the published DEMs is $<0.5 \mathrm{~m}$. Based on this comparison, we conclude that topographic information extracted from ASP models is not significantly different from published models generated using the closed source, proprietary SOCET SET tool.

\section{B1. Preprocessing of image pairs}

Using the Integrated Software for Imagers and Spectrometers (ISIS 3.4) developed by the United States Geological Survey (Anderson et al. [2004], Becker et al. [2007]), HiRISE experimental data records were mosaicked and map-projected. ISIS was then used to normalize and equalize the raw stereo pair images in tandem to optimize the results in the later stereo correlation step (see Section B2) when common features are identified automatically (Broxton et al. [2011]). The region around individual craters was cropped using ISIS to reduce total computation time by decreasing the total area processed in the stereo correlation step. The positions of individual craters, as well as an approximate radius, were selected manually using in-house Python scripts. ISIS was then used to crop both images at two sizes. The smaller of these is a square measuring two 
Table 6. Definition of the crater modification index $\left(i_{M}\right)$, based on visual inspection of HiRISE and THEMIS night-IR imagery. The modification index $i_{\mathrm{M}}$ is computed by summing all applicable scores. Note that some criteria are mutually exclusive while others are not: e.g., a crater cavity cannot simultaneously have a large and small fill deposit, but ejecta may be well-preserved and retain rays. The modification index has one of nine values, ranging from -2 to +6 , where -2 implies exceptionally good preservation.

\begin{tabular}{|c|c|c|c|}
\hline Score & Summary & Source & Observation \\
\hline-1 & ejecta retain rays & $\begin{array}{l}\text { HiRISE, } \\
\text { THEMIS }\end{array}$ & $\begin{array}{l}\text { Rayed distal ejecta are visible via textural or } \\
\text { brightness contrast with surroundings. }\end{array}$ \\
\hline-1 & ejecta are well-preserved & $\begin{array}{l}\text { HiRISE, } \\
\text { THEMIS }\end{array}$ & $\begin{array}{l}\text { Ejecta are well-defined and extensive (usually be- } \\
\text { yond } 1 \text { crater radius from rim), visible via textural } \\
\text { or brightness contrast, and are not significantly } \\
\text { buried, scalloped, topographically smoothed, or } \\
\text { otherwise significantly degraded. Distal rays, if } \\
\text { present, may be patchy. }\end{array}$ \\
\hline+1 & ejecta are degraded & $\begin{array}{l}\text { HiRISE, } \\
\text { THEMIS }\end{array}$ & $\begin{array}{l}\text { Ejecta are visible either by textural or brightness } \\
\text { contrast, but are significantly degraded: partially } \\
\text { buried, eroded, scalloped at margins, patchy, or } \\
\text { topographically smoothed. }\end{array}$ \\
\hline+2 & ejecta are not visible & $\begin{array}{l}\text { HiRISE, } \\
\text { THEMIS }\end{array}$ & $\begin{array}{l}\text { Ejecta are not visible via textural or brightness } \\
\text { contrast with surroundings. }\end{array}$ \\
\hline+1 & small cavity deposit & HiRISE & $\begin{array}{l}\text { Crater cavity contains sediment fill or other de- } \\
\text { posit that encompasses between } 25 \% \text { and } 50 \% \text { of } \\
\text { cavity planform area. }\end{array}$ \\
\hline+2 & large cavity deposit & HiRISE & $\begin{array}{l}\text { Cavity contains sediment fill or other deposit that } \\
\text { encompasses }>50 \% \text { of cavity planform area. }\end{array}$ \\
\hline+1 & lightly cratered & HiRISE & $\begin{array}{l}\text { Between } 1 \text { and } 5 \text { craters visible inside or within } \\
1 \text { crater radius of rim or on continuous ejecta } \\
\text { (whichever is smaller), when crater is viewed at } \\
350 \mathrm{px} / \text { diameter. }\end{array}$ \\
\hline+2 & heavily cratered & HiRISE & $\begin{array}{l}\text { More than } 5 \text { craters visible inside or within } 1 \\
\text { crater radius of rim or on continuous ejecta } \\
\text { (whichever is smaller), when crater is viewed at } \\
350 \mathrm{px} / \text { diameter. }\end{array}$ \\
\hline
\end{tabular}

crater diameters on a side, while the larger measures 10 crater diameters on a side. The former is called the cavity model, used to measure properties of the cavity and rim shape, while the latter context model is used to measure rim height and the average elevation of the surrounding terrain.

To reduce total computation time in the stereo correlation step, cropped images exceeding a threshold number of pixels $n$ on a side were reduced to a dimension of $n \times n$ pixels using ISIS, where $n=1500$ or 3000 pixels. (Early in the project, $n=1500$ was used until superior computing resources became available; images were downsampled to $n=1500$ for $13 \%$ of craters in the database.) As a result, the cropped elevation models have a range of resolutions, and the context models in many cases have a lower resolution than the cavity models. Apart from the manual selection of craters and radii, the pre-processing steps were automated using terminal shell scripts (bash), modeled on the examples in Broxton et al. [2011].

The map-projected images used to generate our models were not bundle-adjusted because we are not concerned with the absolute position and orientation of the craters, and because the craters are too small to be affected by a slight warping that sometimes but rarely re- sults from omitting the bundle-adjustment step (Broxton et al. [2011]).

\section{B2. Stereo correlation}

The Ames Stereo Pipeline 2.0 (ASP) was used to compute the correlation of pixel neighborhoods in each image in order to generate disparity maps and digital elevation models (Broxton and Edwards [2008], Moratto et al. [2010]). The projection used was equirectangular in all cases, with center of the projection equal to centerof-image latitude. We used the default parameters recommended in Broxton et al. [2011] except in cases where the automatically-selected disparity search range was obviously too small, signaled by a crater whose floor contained no valid elevations. These cases were reprocessed using a larger, prescribed disparity search range. Because elevations in a stereo-derived DEM indicate the elevation of features that are resolved by pixel neighborhoods rather than single pixels, the size of the final models was reduced so that post spacing is four image pixels (3 or 4 pixels is conventional: e.g., Broxton et al. [2011], Kirk et al. [2008], Heipke et al. [2007]). Because of the image downsampling described earlier, the resulting resolution of our models ranged from roughly 1 
$\mathrm{m} / \mathrm{px}$ for the smallest craters to approximately $15 \mathrm{~m} / \mathrm{px}$ for the largest craters.

\section{B3. Post-processing of ASP-derived DEMs}

The ASP also generates maps of triangulation error, which it uses to eliminate pixels with neighborhoods that cannot be matched with confidence to any region in the stereo partner. Our in-house post-processing script applied three additional steps to automatically remove pixels with elevations that are likely erroneous. These steps were developed on a trial-and-error basis in order to eliminate discontinuities and anomalous elevations that had no clear basis in the original stereo images. (1) Pixels bounding a slope that exceeds a grade of $700 \%$ were purged (set equal to a null value). We found that this commonly has the effect of isolating regions of spurious elevations. (2) Pixels with an ASP-reported triangulation error in excess of two standard deviations from the average triangulation error were also purged. (3) Pixels belonging to islands of elevation data (surrounded by null values) were then also removed. We also found in a minority of cases that shadows near crater rims led to an elevation artifact resembling a ledge or bench. In these cases we applied a manuallyoutlined shadow mask. The DEM resulting from these steps is called the filtered DEM.

From the filtered DEM we generate the interpolated DEM using an inpainting method. The majority of shape characters described in this paper were measured from the filtered DEM rather than the interpolated DEM: only the cavity volume and the cavity contour were derived from the interpolated DEM.

\section{B4. SOCET SET and ASP DEM comparison}

The Ames Stereo Pipeline (ASP) is a relatively new tool that has already been used in a number of studies to generate elevation models from orbiter and rover imagery (e.g., McCoy et al. [2008], Öhman and Kring [2012], Harrison et al. [2013], Peel and Fassett [2013]). So far, there has been little work to attempt to validate ASP-derived products (Laura et al. [2012]) in the way that has been accomplished for the intermediateresolution HRSC DEMs (Heipke et al. [2007]). The ASP has the advantage of being free and open source software that can be examined and modified by the scientific community. The process used to create the DEMs published by the HiRISE Science Team (213 as of 6 Oct 2014) is described in Kirk et al. [2008] and makes use of the free and open source USGS Integrated System for Imagers and Spectrometers (ISIS) for stereo image projection and radiometric calibration, and then the non-free, proprietary, and closed-source SOCET SET by BAE Systems to compute the disparity map and elevation model.

Because HiRISE provides the highest-possible resolution of any orbiter camera, elevation models derived from HiRISE cannot be validated by comparison with models at comparable or higher resolution except by using rover imagery, which is invariably confined to a small region (Kirk et al. [2008]). Even this comparison is made difficult by the inaccuracies of rover localization that affect the stitching together of wide-baseline observations ( Li et al. [2007]). HRSC DEMs generated using a variety of techniques have been compared to MOLA track elevations (Heipke et al. [2007]) but MOLA spots are too widely-spaced and their footprints are too large to support a validation of topography over the spatial scales addressed in this paper. We therefore take the approach of comparing our post-processed ASP-derived DEMs with a subset of the models published by the HiRISE Science Team.

We generated models of 20 impact craters from twelve HiRISE stereo pairs. All craters also occur in DEMs published by the HiRISE Science Team. Their locations, diameters, and the model resolutions are listed in Table 7 . The published models were resized to match the resolution of our models in the case of large craters where we used downsampled images. Only four craters in this set occur in the main study, as most of these craters are significantly modified. For these comparisons, we performed no manual editing of the ASP-derived DEMs (no shadow-masking).

To compare models, we first automatically locate the rim and geometric center of each crater using the methods described in Section 3.1. A plane is fitted to the rim traces and subtracted from each model so that elevations can be compared. We then sample 360 evenlyspaced radial profiles for each model and compare each one to its counterpart by subtracting one from the other and then computing the average absolute difference. The radial position of each profile is shifted with respect to its counterpart in the comparison model until the average difference is minimized (because model differences cause discrepancies in the rim topography and centroid position). The minimized average difference between radial profiles in the ASP and SOCET SETderived models (averaged over 360 profiles) is reported as $\bar{\Delta} z$ in Table 7 . Example radial profile comparisons with elevation differences that are typical of the crater as a whole are plotted in Figures 16 through 18.

We find that for small craters $(D<500 \mathrm{~m})$, it is usually the case that $\overline{\Delta z} \leq 0.5 \mathrm{~m}$. For larger craters, the average difference between the models climbs to slightly greater than $1 \mathrm{~m}$. We suggest this is largely because of discrepancies between the rim traces that determine the fitted rim planes, confounding the comparison of elevations and adding systematic offsets. The larger the crater, the greater the chance of a discrepancy in the rim trace, which leads to a larger systematic elevation offset. We conclude that the elevation for small craters, that can be more accurately localized for model comparisons, supply a more typical average elevation discrepancy of $\sim 0.5 \mathrm{~m}$ or less. 
Table 7. Results for comparison of ASP-derived elevation models and published HiRISE Science Team models (created using SOCET SET, following Kirk et al. [2008]) of 20 craters (IDs "a" through "t"). The quantity $\bar{\Delta} z$ indicates the average difference between elevations from both kinds of models, measured along 360 radial profiles. Also reported are the standard deviations of this average among the 360 profiles, along with DEM resolution for the ASP model $\left(\delta s_{\text {asp }}\right)$ and published HiRISE model ( $\delta s_{\mathrm{soc}}$, after any resizing), latitude and longitude of each crater, HiRISE observation IDs of the stereo pair images, and the location of the crater as a fraction of the map-projected image from the left $\left(f_{\mathrm{x}}\right)$ and down from the northward top of the image $\left(f_{\mathrm{y}}\right)$.

\begin{tabular}{|c|c|c|c|c|c|c|c|c|c|c|}
\hline ID & $D[\mathrm{~m}]$ & lon. & lat. & $\Delta z[\mathrm{~m}]$ & Observation ID 1 & Observation ID 2 & $\mathrm{f}_{\mathrm{x}}$ & $\mathrm{f}_{\mathrm{y}}$ & $\delta s_{\text {asp }}[\mathrm{m}]$ & $\delta s_{\mathrm{soc}}[\mathrm{m}]$ \\
\hline$\overline{\mathrm{a}}$ & $199 \pm 13$ & $222.3614^{\circ}$ & $21.4659^{\circ}$ & $0.31 \pm 0.07$ & PSP_001432_2015 & PSP_001630_2015 & 0.59 & 0.91 & 1.13 & 1.01 \\
\hline $\mathrm{b}$ & $206 \pm 6$ & $339.5011^{\circ}$ & $25.0636^{\circ}$ & $0.53 \pm 0.21$ & PSP_006887_2050 & PSP_007823_2050 & 0.25 & 0.05 & 1.17 & 1.01 \\
\hline $\mathrm{c}$ & $123 \pm 8$ & $162.9139^{\circ}$ & $1.2976^{\circ}$ & $0.20 \pm 0.08$ & PSP_003874_1815 & PSP_004375_1815 & 0.42 & 0.34 & 1.16 & 1.01 \\
\hline d & $135 \pm 8$ & $55.4782^{\circ}$ & $-38.7349^{\circ}$ & $0.49 \pm 0.28$ & PSP_010563_1410 & PSP_006278_1410 & 0.52 & 0.36 & 1.88 & 2.01 \\
\hline e & $134 \pm 12$ & $162.9006^{\circ}$ & $1.3625^{\circ}$ & $0.21 \pm 0.08$ & PSP_003874_1815 & PSP_004375_1815 & 0.30 & 0.07 & 1.17 & 1.01 \\
\hline$f$ & $67 \pm 3$ & $226.9586^{\circ}$ & $-28.6257^{\circ}$ & $0.26 \pm 0.07$ & PSP_002118_1510 & PSP_003608_1510 & 0.67 & 0.33 & 0.98 & 1.01 \\
\hline $\mathrm{g}$ & $239 \pm 10$ & $205.4925^{\circ}$ & $-33.4931^{\circ}$ & $0.59 \pm 0.17$ & PSP_009318_1465 & ESP_017243_1465 & 0.59 & 0.82 & 1.92 & 2.01 \\
\hline $\mathrm{h}$ & $270 \pm 11$ & $303.4579^{\circ}$ & $21.3560^{\circ}$ & $0.34 \pm 0.13$ & PSP_003460_2015 & PSP_003605_2015 & 0.20 & 0.28 & 1.13 & 1.01 \\
\hline i & $346 \pm 14$ & $175.5491^{\circ}$ & $-14.6608^{\circ}$ & $0.31 \pm 0.20$ & PSP_001513_1655 & PSP_001777_1650 & 0.86 & 0.68 & 1.06 & 1.01 \\
\hline j & $232 \pm 11$ & $175.4749^{\circ}$ & $-14.5673^{\circ}$ & $0.15 \pm 0.05$ & PSP_001513_1655 & PSP_001777_1650 & 0.33 & 0.43 & 1.05 & 1.01 \\
\hline $\mathrm{k}$ & $321 \pm 13$ & $175.4780^{\circ}$ & $-14.5241^{\circ}$ & $0.32 \pm 0.14$ & PSP_001513_1655 & PSP_001777_1650 & 0.35 & 0.32 & 1.05 & 1.01 \\
\hline 1 & $248 \pm 13$ & $137.2876^{\circ}$ & $-4.5638^{\circ}$ & $0.22 \pm 0.10$ & PSP_009650_1755 & PSP_009716_1755 & 0.35 & 0.39 & 1.07 & 1.01 \\
\hline $\mathrm{m}$ & $404 \pm 15$ & $137.3402^{\circ}$ & $-4.5839^{\circ}$ & $0.41 \pm 0.20$ & PSP_009650_1755 & PSP_009716_1755 & 0.71 & 0.43 & 1.08 & 1.01 \\
\hline $\mathrm{n}$ & $1147 \pm 25$ & $303.5394^{\circ}$ & $21.2597^{\circ}$ & $0.68 \pm 0.31$ & PSP_003460_2015 & PSP_003605_2015 & 0.74 & 0.53 & 2.86 & 2.37 \\
\hline $\mathrm{o}$ & $881 \pm 36$ & $339.9992^{\circ}$ & $23.3105^{\circ}$ & $1.00 \pm 0.62$ & ESP_022288_2035 & ESP_022354_2035 & 0.49 & 0.88 & 2.07 & 1.78 \\
\hline $\mathrm{p}$ & $2000 \pm 77$ & $205.4952^{\circ}$ & $-33.3585^{\circ}$ & $1.78 \pm 0.75$ & PSP_009318_1465 & ESP_017243_1465 & 0.61 & 0.22 & 5.02 & 4.15 \\
\hline q & $816 \pm 30$ & $354.4995^{\circ}$ & $-2.0540^{\circ}$ & $0.59 \pm 0.14$ & PSP_001414_1780 & PSP_001612_1780 & 0.50 & 0.58 & 2.12 & 1.54 \\
\hline r & $671 \pm 24$ & $137.2780^{\circ}$ & $-4.5074^{\circ}$ & $0.26 \pm 0.10$ & PSP_009650_1755 & PSP_009716_1755 & 0.28 & 0.25 & 1.61 & 1.25 \\
\hline s & $2494 \pm 41$ & $209.7043^{\circ}$ & $16.4248^{\circ}$ & $1.37 \pm 0.36$ & PSP_005837_1965 & PSP_005903_1965 & 0.43 & 0.48 & 6.52 & 5.22 \\
\hline $\mathrm{t}$ & $2917 \pm 60$ & $226.9308^{\circ}$ & $-28.6806^{\circ}$ & $3.08 \pm 1.52$ & PSP_002118_1510 & PSP_003608_1510 & 0.46 & 0.48 & 7.11 & 5.76 \\
\hline
\end{tabular}

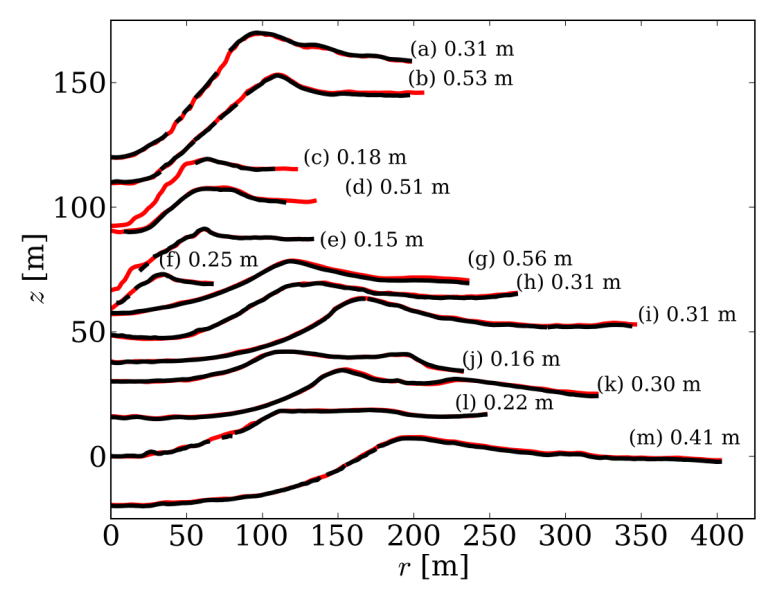

Figure 16. Comparison of radial profiles extracted from elevation models generated using the process described in this paper (black) and from models published by the HiRISE Science Team and generated using SOCET SET (red). The average difference between the profiles is reported at the right, while the average for the crater as a whole is reported in Table 7 . This figure illustrates the results for craters in the smallest size range $(D<500 \mathrm{~m})$. See Figures 17 and 18 for the large-crater comparisons.

We conclude that our elevation models are in close agreement with the published models, and that our approach for generating elevation models is appropriate for morphometric analyses like the one presented in this study: i.e., in which the absolute (as opposed to rela-

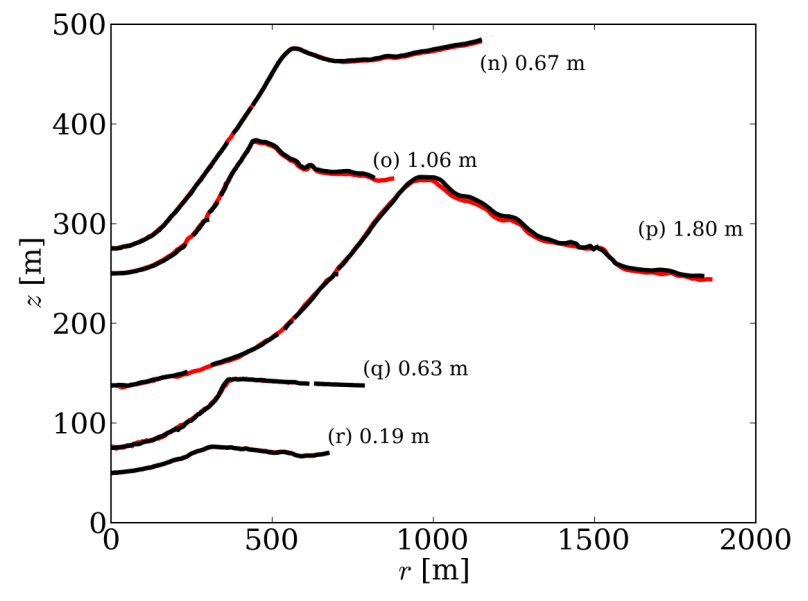

Figure 17. Elevation profiles extracted from ASP- and SOCET SET-derived DEMs (black and red, respectively) for craters of intermediate size. See Section B4 and the Figure 16 caption for details.

tive) elevation and orientation of topography is not important.

Acknowledgments. We gratefully acknowledge grants from Wellesley College alumnae funds to support the work of L.G. (Schiff fellowship), R.G., and M.F. (Malone Summer Research Grant). We are grateful to Arden Radford (Wellesley) for assistance with identifying the craters addressed in this study, and Trent Hare (USGS) for GIS-related advice and for providing a raster version of the Tanaka et al. [2014] map. We also thank Nadine Barlow and Stuart Robbins for very helpful reviews that have significantly improved the manuscript. All of the work presented in this paper was accomplished using free and open source 


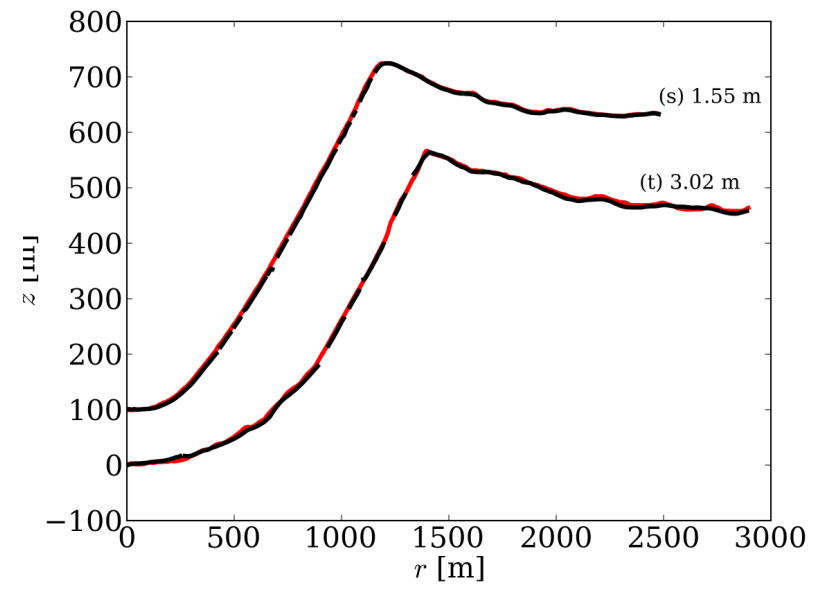

Figure 18. Elevation profiles extracted from ASP- and SOCET SET-derived DEMs (black and red, respectively) for two craters with $D>2 \mathrm{~km}$. See Section B4 and the Figure 16 caption for details.

software: we are grateful to the developers of the Ames Stereo Pipeline, GNU core utilities, Emacs, Inkscape, QGIS, Python, Scientific and Numerical Python, Matplotlib, LATEX, and Debian GNU/Linux. A database containing the quantities used to produce the tables and graphs in this paper are available for download as supplementary materials from JGR Planets. Readers may also contact the corresponding author (wwatters@wellesley.edu) to request a copy of these data.

\section{References}

Anderson, J. A., S. C. Sides, D. L. Soltesz, T. L. Sucharski, and K. J. Becker (2004), Modernization of the Integrated Software for Imagers and Spectrometers, in Lunar and Planetary Institute Science Conference Abstracts, vol. 35, edited by S. Mackwell and E. Stansbery, p. 2039.

Baker, D. M. H., J. W. Head, G. A. Neumann, D. E. Smith, and M. T. Zuber (2012), The transition from complex craters to multi-ring basins on the Moon: Quantitative geometric properties from Lunar Reconnaissance Orbiter Lunar Orbiter Laser Altimeter (LOLA) data, Journal of Geophysical Research: Planets, 117, E00H16, doi:10.1029/2011JE004021.

Banks, M. E., S. Byrne, K. Galla, A. S. McEwen, V. J. Bray, C. M. Dundas, K. E. Fishbaugh, K. E. Herkenhoff, and B. C Murray (2010), Crater population and resurfacing of the Martian north polar layered deposits, Journal of Geophysical Research: Planets, 115, E08006, doi:10.1029/2009JE003523.

Barlow, N. G. (2004), Martian subsurface volatile concentrations as a function of time: Clues from layered ejecta craters, Geophysical Research Letters, 31, L05703, doi: 10.1029/2003GL019075.

Barlow, N. G., J. M. Boyce, F. M. Costard, R. A. Craddock, J. B. Garvin, S. E. Sakimoto, R. O. Kuzmin, D. J. Roddy, and L. A Soderblom (2000), Standardizing the nomenclature of martian impact crater ejecta morphologies, Journal of Geophysical Research: Planets (1991-2012), 105 (E11), 26,733-26,738.

Barnouin, O. S., M. T. Zuber, D. E. Smith, G. A. Neumann, R. R. Herrick, J. E. Chappelow, S. L. Murchie, and L. M. Prockter (2012), The morphology of craters on Mercury: Results from MESSENGER flybys, Icarus, 219, 414-427, doi: 10.1016/j.icarus.2012.02.029.

Barnouin-Jha, O. S., S. Baloga, and L. Glaze (2005), Comparing landslides to fluidized crater ejecta on Mars, Journal of Geophysical Research: Planets, 110, E04010, doi: 10.1029/2003JE002214.

Basilevsky, A., M. Kreslavsky, I. Karachevtseva, and E. Gusakova (2014), Morphometry of small impact craters in the Lunokhod1 and Lunokhod-2 study areas, Planetary and Space Science, 92, 77-87, doi:http://dx.doi.org/10.1016/j.pss.2013.12.016.
Becker, K. J., J. A. Anderson, S. C. Sides, E. A. Miller, E. M. Eliason, and L. P. Keszthelyi (2007), Processing HiRISE Images Using ISIS3, in Lunar and Planetary Institute Science Conference Abstracts, vol. 38, p. 1779.

Beyer, R. A., B. Archinal, Y. Chen, K. Edmundson, D. Harbour, E. Howington-Kraus, R. Li, A. McEwen, S. Mattson, Z. Moratto, J. Oberst, M. Rosiek, F. Scholten, T. Tran, M. Robinson, and LROC Team (2010), LROC Stereo Data Results of Initial Analysis, in Lunar and Planetary Institute Science Conference Abstracts, vol. 41, p. 2678.

Black, B. A., and S. T. Stewart (2008), Excess ejecta craters record episodic ice-rich layers at middle latitudes on Mars, Journal of Geophysical Research: Planets, 113, E02015, doi: 10.1029/2007JE002888.

Boyce, J. M., and H. Garbeil (2007), Geometric relationships of pristine Martian complex impact craters, and their implications to Mars geologic history, Geophysical Research Letters, 34, L16201, doi:10.1029/2007GL029731.

Boyce, J. M., and P. J. Mouginis-Mark (2006), Martian craters viewed by the thermal emission imaging system instrument: Double-layered ejecta craters, Journal of Geophysical Research: Planets, 111(E10), doi:10.1029/2005JE002638.

Boyce, J. M., P. Mouginis-Mark, and H. Garbeil (2005), Ancient oceans in the northern lowlands of Mars: Evidence from impact crater depth/diameter relationships, Journal of Geophysical Research: Planets, 110, E03008, doi: 10.1029/2004JE002328.

Boyce, J. M., P. Mouginis-Mark, H. Garbeil, and L. L. Tornabene (2006), Deep impact craters in the Isidis and southwestern Utopia Planitia regions of Mars: High target material strength as a possible cause, Geophys. Res. Lett., 33, L06202, doi:10.1029/2005GL024462.

Boynton, W. V., W. C. Feldman, S. W. Squyres, T. H. Prettyman, J. Brckner, L. G. Evans, R. C. Reedy, R. Starr, J. R. Arnold, D. M. Drake, P. A. J. Englert, A. E. Metzger, I. Mitrofanov, J. I. Trombka, C. d'Uston, H. Wnke, O. Gasnault, D. K. Hamara, D. M. Janes, R. L. Marcialis, S. Maurice, I. Mikheeva, G. J. Taylor, R. Tokar, and C. Shinohara (2002), Distribution of hydrogen in the near surface of Mars: Evidence for subsurface ice deposits, Science, 297(5578), 81-85, doi:10.1126/science.1073722.

Broxton, M., R. Beyer, Z. Moratto, M. Lundy, and K. Husmann (2011), The Ames Stereo Pipeline: NASA's Open Source Automated Stereogrammetry Software (user manual), NASA NeoGeography Toolkit.

Broxton, M. J., and L. J. Edwards (2008), The Ames Stereo Pipeline: Automated 3D Surface Reconstruction from Orbital Imagery, in Lunar and Planetary Institute Science Conference Abstracts, vol. 39, p. 2419.

Byrne, S., C. M. Dundas, M. R. Kennedy, M. T. Mellon, A. S. McEwen, S. C. Cull, I. J. Daubar, D. E. Shean, K. D. Seelos, S. L. Murchie, et al. (2009), Distribution of midlatitude ground ice on mars from new impact craters, Science, 325 (5948), 1674-1676.

Carson, M. A., and M. J. Kirkby (1972), Hillslope form and process, vol. 475, Cambridge University Press Cambridge.

Christensen, P., B. Jakosky, H. Kieffer, M. Malin, J. McSween, HarryY., K. Nealson, G. Mehall, S. Silverman, S. Ferry, M. Caplinger, and M. Ravine (2004), The Thermal Emission Imaging System (THEMIS) for the Mars 2001 Odyssey Mission, Space Science Reviews, 110(1-2), 85-130, doi:10.1023/B:SPAC.0000021008.16305.94.

Conway, S. J., and N. Mangold (2013), Evidence for Amazonian mid-latitude glaciation on Mars from impact crater asymmetry, Icarus, 225, 413-423, doi:10.1016/j.icarus.2013.04.013.

Craddock, R. A., T. A. Maxwell, and A. D. Howard (1997), Crater morphometry and modification in the Sinus Sabaeus and Margaritifer Sinus regions of Mars, Journal of Geophysical Research, 102, 13,321-13,340, doi:10.1029/97JE01084.

Daubar, I. J., A. S. McEwen, S. Byrne, M. R. Kennedy, B. Ivanov (2013), The current Martian cratering rate., Icarus, 225, 1,506-516, doi:10.1016/j.icarus.2013.04.009.

Fagan, A. L., and S. E. H. Sakimoto (2012), An investigation of Martian northern high-latitude and polar impact crater interiors: Atypical interior topographic features and cavity wall slopes, Meteoritics and Planetary Science, 47, 970-991, doi: 10.1111/j.1945-5100.2012.01365.x. 
Fassett, C. I., and B. J. Thomson (2014), Crater degradation on the lunar maria: Topographic diffusion and the rate of erosion on the moon, Journal of Geophysical Research: Planets, 119, 2255-2271, doi:10.1002/2014JE004698.

Forsberg-Taylor, N. K., A. D. Howard, and R. A. Craddock (2004), Crater degradation in the Martian highlands: Morphometric analysis of the Sinus Sabaeus region and simulation modeling suggest fluvial processes, Journal of Geophysical Research: Planets, 109, E05002, doi:10.1029/2004JE002242.

Fudali, R., D. Milton, K. Fredriksson, and A. Dube (1980), Morphology of Lonar crater, India: Comparisons and implications, The Moon and the Planets, 23(4), 493-515.

Garvin, J. B., and J. J. Frawley (1998), Geometric properties of Martian impact craters: Preliminary results from the Mars Orbiter Laser Altimeter, Geophys. Res. Lett., 25, 4405-4408.

Garvin, J. B., S. E. H. Sakimoto, J. J. Frawley, and C. Schnetzler (2000), North polar region craterforms on Mars: Geometric characteristics from the Mars Orbiter Laser Altimeter, Icarus, $144,329-352$.

Garvin, J. B., S. E. H. Sakimoto, and J. J. Frawley (2003), Craters on Mars: Global Geometric Properties from Gridded MOLA Topography, in Sixth International Conference on Mars, edited by A. L. Albee and H. H. Kieffer, p. 3277.

Geiger, L. M. (2013), Statistical analysis of simple martian impact crater morphometry, Undergraduate honors thesis; http://repository.wellesley.edu/thesiscollection/136/, Wellesley College, 106 Central Street, Wellesley, MA 02481.

Geissler, P. E., I. J. Daubar, A. S. McEwen, N. T. Bridges, and C. M. Dundas (2010), Eolian Degradation of Young Martian Craters, in Lunar and Planetary Institute Science Conference Abstracts, vol. 41, p. 2591.

Grant, J. A. (1999), Evaluating the evolution of process specific degradation signatures around impact craters, International Journal of Impact Engineering, 23(1, Part 1), 331 - 340, doi: http://dx.doi.org/10.1016/S0734-743X(99)00084-6.

Grant, J. A., R. E. Arvidson, L. S. Crumpler, M. P. Golombek, B. Hahn, A. F. C. Haldemann, R. Li, L. A. Soderblom, S. W. Squyres, S. P. Wright, and W. A. Watters (2006), Crater gradation in Gusev crater and Meridiani Planum, Mars., Journal of Geophysical Research: Planets, 111.

Harrison, S. K., M. R. Balme, A. Hagermann, J. B. Murray, J.-P. Muller, and A. Wilson (2013), A branching, positive relief network in the middle member of the Medusae Fossae Formation, equatorial Mars-Evidence for sapping?, Planet. Space Sci., 85, 142-163, doi:10.1016/j.pss.2013.06.004.

Heipke, C., J. Oberst, J. Albertz, M. Attwenger, P. Dorninger, E. Dorrer, M. Ewe, S. Gehrke, K. Gwinner, H. Hirschmüller, J. R. Kim, R. L. Kirk, H. Mayer, J.-P. Muller, R. Rengarajan, M. Rentsch, R. Schmidt, F. Scholten, J. Shan, M. Spiegel, M. Wählisch, G. Neukum, and the HRSC CoInvestigator Team (2007), Evaluating planetary digital terrain models-The HRSC DTM test, Planetary and Space Science, 55, 2173-2191, doi:10.1016/j.pss.2007.07.006.

Herrick, R., V. Sharpton, M. Malin, S. Lyons, and K. Feely (1997), Morphology and morphometry of impact craters, in Venus II: Geology, Geophysics, Atmosphere, and Solar Wind Environment, vol. 1, pp. 1015-1046, Tucson, University of Arizona Press.

Howard, A. D. (2004), Simple Non-fluvial Models of Planetary Surface Modification, with Application to Mars, in Lunar and Planetary Institute Science Conference Abstracts, vol. 35, edited by S. Mackwell and E. Stansbery, p. 1054.

Jankowski, D., and S. Squyres (1993), "Softened" Impact Craters on Mars: Implications for Ground Ice and the Structure of the Martian Megaregolith, Icarus, 106 (2), 365-379.

Jaumann, R., G. Neukum, T. Behnke, T. C. Duxbury, K. Eichentopf, J. Flohrer, S. v. Gasselt, B. Giese, K. Gwinner, E. Hauber, H. Hoffmann, A. Hoffmeister, U. Köhler, K.D. Matz, T. B. McCord, V. Mertens, J. Oberst, R. Pischel, D. Reiss, E. Ress, T. Roatsch, P. Saiger, F. Scholten, G. Schwarz, K. Stephan, M. Wählisch, and the HRSC CoInvestigator Team (2007), The high-resolution stereo camera (HRSC) experiment on Mars Express: Instrument aspects and experiment conduct from interplanetary cruise through the nominal mission, Planet. Space Sci., 55, 928-952, doi: 10.1016/j.pss.2006.12.003.
Kalynn, J., C. L. Johnson, G. R. Osinski, and O. Barnouin (2013), Topographic characterization of lunar complex craters, Geophysical Research Letters, 40, 38-42, doi: 10.1029/2012GL053608.

Kirk, R. L., E. Howington-Kraus, M. R. Rosiek, J. A. Anderson, B. A. Archinal, K. J. Becker, D. A. Cook, D. M. Galuszka, P. E. Geissler, T. M. Hare, I. M. Holmberg, L. P. Keszthelyi, B. L. Redding, W. A. Delamere, D. Gallagher, J. D. Chapel, E. M. Eliason, R. King, and A. S. McEwen (2008), Ultrahigh resolution topographic mapping of Mars with MRO HiRISE stereo images: Meter-scale slopes of candidate Phoenix landing sites, Journal of Geophysical Research: Planets, 113, E00A24, doi:10.1029/2007JE003000.

Kreslavsky, M. A., and J. W. Head (2006), Modification of impact craters in the northern plains of Mars: Implications for Amazonian climate history, Meteoritics and Planetary Science, 41, 1633-1646, doi:10.1111/j.1945-5100.2006.tb00441.x.

Laura, J. R., D. Miller, and M. V. Paul (2012), AMES Stereo Pipeline Derived DEM Accuracy Experiment Using LROCNAC Stereopairs and Weighted Spatial Dependence Simulation for Lunar Site Selection, in Lunar and Planetary Institute Science Conference Abstracts, vol. 43, p. 2371.

Li, R., R. E. Arvidson, M. G. K. Di, J. Guinn, A. Johnson, M. Maimone, L. H. Matthies, M. Malin, T. Parker, S. W. Squyres, and W. A. Watters (2007), Opportunity rover localization and topographic mapping at the landing site of Meridiani Planum, Mars, Journal of Geophysical Research: Planets, 112, doi:10.1029/2006JE002776.

McCoy, T. J., M. Sims, M. E. Schmidt, L. Edwards, L. L. Tornabene, L. S. Crumpler, B. A. Cohen, L. A. Soderblom, D. L. Blaney, S. W. Squyres, R. E. Arvidson, J. W. R. Jr., E. Treguier, C. dUston, J. A. Grant, . H. Y. McSween Jr. M. P. Golombek, 7 A. F. C. Haldemann, and . P. A. de Souza Jr.15 (2008), Structure, stratigraphy, and origin of Husband Hill, Columbia Hills, Gusev Crater, Mars, Journal of Geophysical Research: Planets, 113, E06S03, doi:10.1029/2007JE003041.

McEwen, A. S., B. S. Preblich, E. P. Turtle, N. A. Artemieva, and M. P. Golombek (2005), The rayed crater Zunil and interpretations of small impact craters on Mars, Icarus, 176, 351-381.

McEwen, A. S., E. M. Eliason, J. W. Bergstrom, N. T. Bridges, C. J. Hansen, W. A. Delamere, J. A. Grant, V. C. Gulick, K. E. Herkenhoff, L. Keszthelyi, R. L. Kirk, M. T. Mellon, S. W. Squyres, N. Thomas, and C. M. Weitz (2007), Mars Reconnaissance Orbiter's High Resolution Imaging Science Experiment (HiRISE), Journal of Geophysical Research: Planets, 112, E05S02, doi:10.1029/2005JE002605.

Moratto, Z. M., M. J. Broxton, R. A. Beyer, M. Lundy, and K. Husmann (2010), Ames Stereo Pipeline, NASA's Open Source Automated Stereogrammetry Software, in Lunar and Planetary Institute Science Conference Abstracts, vol. 41, p. 2364.

Öhman, T., and D. A. Kring (2012), Photogeologic analysis of impact melt-rich lithologies in Kepler crater that could be sampled by future missions, Journal of Geophysical Research: Planets, 117, E00H08, doi:10.1029/2011JE003918.

Peel, S. E., and C. I. Fassett (2013), Valleys in pit craters on Mars: Characteristics, distribution, and formation mechanisms, Icarus, 225, 272-282, doi:10.1016/j.icarus.2013.03.031.

Pike, R. J. (1974), Depth/diameter relations of fresh lunar craters - Revision from spacecraft data, Geophysical Research Letters, 1, 291-294, doi:10.1029/GL001i007p00291.

Pike, R. J. (1977), Size-dependence in the shape of fresh impact craters on the Moon, in Impact and explosion cratering, edited by D. J. Roddy, R. O. Pepin, and R. B. Merrill, pp. 489-509, Pergamon Press, New York, New York.

Pike, R. J. (1980a), Control of crater morphology by gravity and target type - Mars, Earth, Moon, in Lunar and Planetary Science Conference Proceedings, vol. 11, edited by S. A. Bedini, pp. 2159-2189.

Pike, R. J. (1980b), Terrain Dependence of Crater Morphology on Mars: both yes and no, in Lunar and Planetary Institute Science Conference Abstracts, vol. 11, pp. 885-887.

Pike, R. J. (1988), Geomorphology of impact craters on Mercury, pp. 165-273, University of Arizona Press.

Pike, R. J., and P. A. Davis (1984), Toward a Topographic Model of Martian Craters from Photoclinometry, in Lunar and Planetary Institute Science Conference Abstracts, vol. 15, pp. 645646. 
Popova, O., I. Nemtchinov, and W. K. Hartmann (2003), Bolides in the present and past martian atmosphere and effects on cratering processes, Meteoritics \& Planetary Science, 38(6), 905-925, doi:10.1111/j.1945-5100.2003.tb00287.x.

Press, W. H., S. A. Teukolsky, W. T. Vetterling, and B. P. Flannery (1988), Numerical Recipes in C, 2nd ed., Cambridge Univ. Press, Cambridge, UK.

Robbins, S. J., and B. M. Hynek (2012a), A new global database of Mars impact craters $>1 \mathrm{~km}$ : 2. Global crater properties and regional variations of the simple-to-complex transition diameter, Journal of Geophysical Research: Planets, 117, E06001, doi:10.1029/2011JE003967.

Robbins, S. J., and B. M. Hynek (2012b), A new global database of Mars impact craters $\geq 1 \mathrm{~km}$ : 1 . Database creation, properties, and parameters, Journal of Geophysical Research: Planets, 117, E05004, doi:10.1029/2011JE003966.

Robbins, S. J., and B. M. Hynek (2013), Utility of laser altimeter and stereoscopic terrain models: Application to martian craters, Planetary and Space Science, 86, 57-65, doi: 10.1016/j.pss.2013.06.019.

Robinson, M. S., S. M. Brylow, M. Tschimmel, D. Humm, S. J. Lawrence, P. C. Thomas, B. W. Denevi, E. Bowman-Cisneros, J. Zerr, M. A. Ravine, M. A. Caplinger, F. T. Ghaemi, J. A Schaffner, M. C. Malin, P. Mahanti, A. Bartels, J. Anderson, T. N. Tran, E. M. Eliason, A. S. McEwen, E. Turtle, B. L. Jolliff, and H. Hiesinger (2010), Lunar Reconnaissance Orbiter Camera (LROC) Instrument Overview, Space Sci. Rev., 150, 81-124, doi:10.1007/s11214-010-9634-2.

Senft, L. E., and S. T. Stewart (2008), Impact crater formation in icy layered terrains on Mars, Meteoritics and Planetary Science, 43, 1993-2013, doi:10.1111/j.1945-5100.2008.tb00657.x.

Shoemaker, E. M. (1960), Penetration mechanics of high velocity meteorites, illustrated by Meteor Crater, Arizona, International Geological Congress, 21.

Shoemaker, E. M., and R. E. Eggleton (1961), Terrestrial features of impact origin, Proceedings of the Geophysical Laboratory Lawrence Radiation Cratering Symposium, 1, A1-A27.

Shoemaker, E. M., F. A. MacDonald, and C. S. Shoemaker (2005), Geology of five small Australian impact craters, Aus. J. Earth Sci., 52, 529-544.

Smith, D. E., M. T. Zuber, H. V. Frey, J. B. Garvin, J. W. Head, D. O. Muhleman, G. H. Pettengill, R. J. Phillips, S. C. Solomon, H. J. Zwally, W. B. Banerdt, T. C. Duxbury, M. P. Golombek, F. G. Lemoine, G. A. Neumann, D. D. Rowlands, O. Aharonson, P. G. Ford, A. B. Ivanov, C. L. Johnson, P. J. McGovern, J. B. Abshire, R. S. Afzal, and X. Sun (2001), Mars Orbiter Laser Altimeter: Experiment summary after the first year of global mapping of Mars, J. Geophys. Res., , 106, 23,689-23,722, doi:10.1029/2000JE001364.

Soderblom, L. A. (1970), A model for small-impact erosion applied to the lunar surface, Journal of Geophysical Research 75 (14), 2655-2661, doi:10.1029/JB075i014p02655.

Squyres, S. W., and M. H. Carr (1986), Geomorphic evidence for the distribution of ground ice on Mars, Science, 231, 249-252, doi:10.1126/science.231.4735.249.
Stepinski, T. F., M. P. Mendenhall, and B. D. Bue (2009), Machine cataloging of impact craters on Mars, Icarus, 203, 77-87, doi:10.1016/j.icarus.2009.04.026.

Stewart, S. T., and G. J. Valiant (2006), Martian subsurface properties and crater formation processes inferred from fresh impact crater geometries, Meteor. and Planet. Sci, 41, 15091537.

Stopar, J. D., M. S. Robinson, E. J. Speyerer, K. Burns, H. Gengl, and LROC Team (2012), Regolith Characterization Using LROC NAC Digital Elevation Models of Small Lunar Craters, in Lunar and Planetary Institute Science Conference Abstracts, vol. 43, p. 2729.

Tanaka, K., J. Skinner Jr., J. Dohm, R. Irwin III, E. Kolb, C. Fortezzo, T. Platz, G. Michael, and T. Hare (2014), Geologic map of mars, U.S. Geological Survey Scientific Investigations Maps, (3292).

Tornabene, L. L., J. E. Moersch, H. Y. McSween, A. S. McEwen, J. L. Piatek, K. A. Milam, and P. R. Christensen (2006), Identification of large $(2-10 \mathrm{~km})$ rayed craters on Mars in THEMIS thermal infrared images: Implications for possible Martian meteorite source regions, Journal of Geophysical Research: Planets, 111(E10), E10006, doi:10.1029/2005JE002600.

Turtle, E. P., E. Pierazzo, G. S. Collins, G. R. Osinski, H. J. Melosh, J. V. Morgan, and W. U. Reimold (2005), Impact structures: What does crater diameter mean?, Geological Society of America Special Papers, 384, 25.

Vijayan, S., K. Vani, and S. Sanjeevi (2013), Topographical analysis of lunar impact craters using SELENE images, Advances in Space Research, 52, 1221-1236, doi:10.1016/j.asr.2013.06.025.

Vincent, J.-B., P. Schenk, A. Nathues, H. Sierks, M. Hoffmann, R. Gaskell, S. Marchi, D. O'Brien, M. Sykes, C. Russell, M. Fulchignoni, H. Kellerg, C. Raymond, E. Palmer, and F. Preusker (2013), Crater depth-to-diameter distribution and surface properties of (4) Vesta, Planetary and Space Science, 103, 57-65, doi:10.1016/j.pss.2013.09.003.

Weiss, D. K., and J. W. Head (2014), Ejecta mobility of layered ejecta craters on Mars: Assessing the influence of snow and ice deposits, Icarus, 233(0), 131 - 146, doi: 10.1016/j.icarus.2014.01.038.

Whitehead, J., R. A. F. Grieve, J. K. Garvin, and J. G. Spray (2010), The effects of crater degradation and target differences on the morphologies of Martian complex craters, Geological Society of America Special Papers, 465, 67-81.

Wood, C. A., and L. Anderson (1978), New morphometric data for fresh lunar craters, in Lunar and Planetary Science Conference Proceedings, vol. 9, pp. 3669-3689.

Corresponding author: W. A. Watters; Department of Astronomy, Whitin Observatory, Wellesley College; 106 Central St., Wellesley, MA 02481, USA; (wwatters@wellesley.edu) 